\title{
The north-subducting Rheic Ocean during the Devonian: consequences for the Rhenohercynian ore sites
}

\author{
Jürgen F. von Raumer ${ }^{1}\left[\right.$ $\cdot$ Heinz-Dieter Nesbor ${ }^{2} \cdot$ Gérard M. Stampfli $^{3}$
}

\begin{abstract}
Base metal mining in the Rhenohercynian Zone has a long history. Middle-Upper Devonian to Lower Carboniferous sediment-hosted massive sulfide deposits (SHMS), volcanic-hosted massive sulfide deposits (VHMS) and Lahn-Dill-type iron, and base metal ores occur at several sites in the Rhenohercynian Zone that stretches from the South Portuguese Zone, through the Lizard area, the Rhenish Massif and the Harz Mountain to the Moravo-Silesian Zone of SW Bohemia. During Devonian to Early Carboniferous times, the Rhenohercynian Zone is seen as an evolving rift system developed on subsiding shelf areas of the Old Red continent. A reappraisal of the geotectonic setting of these ore deposits is proposed. The Middle-Upper Devonian to Early Carboniferous time period was characterized by detrital sedimentation, continental intraplate and subduction-related volcanism. The large shelf of the Devonian Old Red continent was the place of thermal subsidence with contemporaneous mobilization of rising thermal fluids along
\end{abstract}

Jürgen F. von Raumer

Juergen.vonraumer@unifr.ch

Heinz-Dieter Nesbor

heinz-dieter.nesbor@hlug.hessen.de

Gérard M. Stampfli

gerard.stampfli@unil.ch

1 Dépt. de Géosciences, Université, 1700 Fribourg, Switzerland

2 Hessisches Landesamt für Naturschutz Organization, Umwelt und Geologie (HLNUG), 65203 Wiesbaden, Germany

3 Earth Sciences Institute (ISTE) Geopolis, UNIL, 1015 Lausanne, Switzerland activated Early Devonian growth faults. Hydrothermal brines equilibrated with the basement and overlying Middle-Upper Devonian detrital deposits forming the SHMS deposits in the southern part of the Pyrite Belt, in the Rhenish Massif and in the Harz areas. Volcanic-hosted massive sulfide deposits (VHMS) formed in the more eastern localities of the Rhenohercynian domain. In contrast, since the Tournaisian period of ore formation, dominant pull-apart triggered magmatic emplacement of acidic rocks, and their metasomatic replacement in the apical zones of felsic domes and sediments in the northern part of the Iberian Pyrite belt, thus changing the general conditions of ore precipitation. This two-step evolution is thought to be controlled by syn- to post-tectonic phases in the Variscan framework, specifically by the transition of geotectonic setting dominated by crustal extension to a one characterized by the subduction of the supposed northern slab of the Rheic Ocean preceding the general Late Variscan crustal shortening and oroclinal bending.

Keywords Base metal and iron ores · Rhenohercynian rift $\cdot$ Rheic Ocean $\cdot$ Subduction-related $\cdot$ Devonian

\section{Introduction}

Comparing the geological zonation of the Variscides (e.g., Franke 2000), Devonian to Lower Carboniferous sediments and volcanics of the Rhenohercynian Zone (Fig. 1) host well-known stratiform ore deposits throughout the European continent, following the Late Variscan oroclinal bending through Western Europe (comp. Martínez Catalán et al. 2012).

Historically, Amstutz et al. (1971, p. 254), interpreting Kräutner's (1970) corresponding map of a continuous metallotect for base metal deposits, quoted "that a geosynclinal 


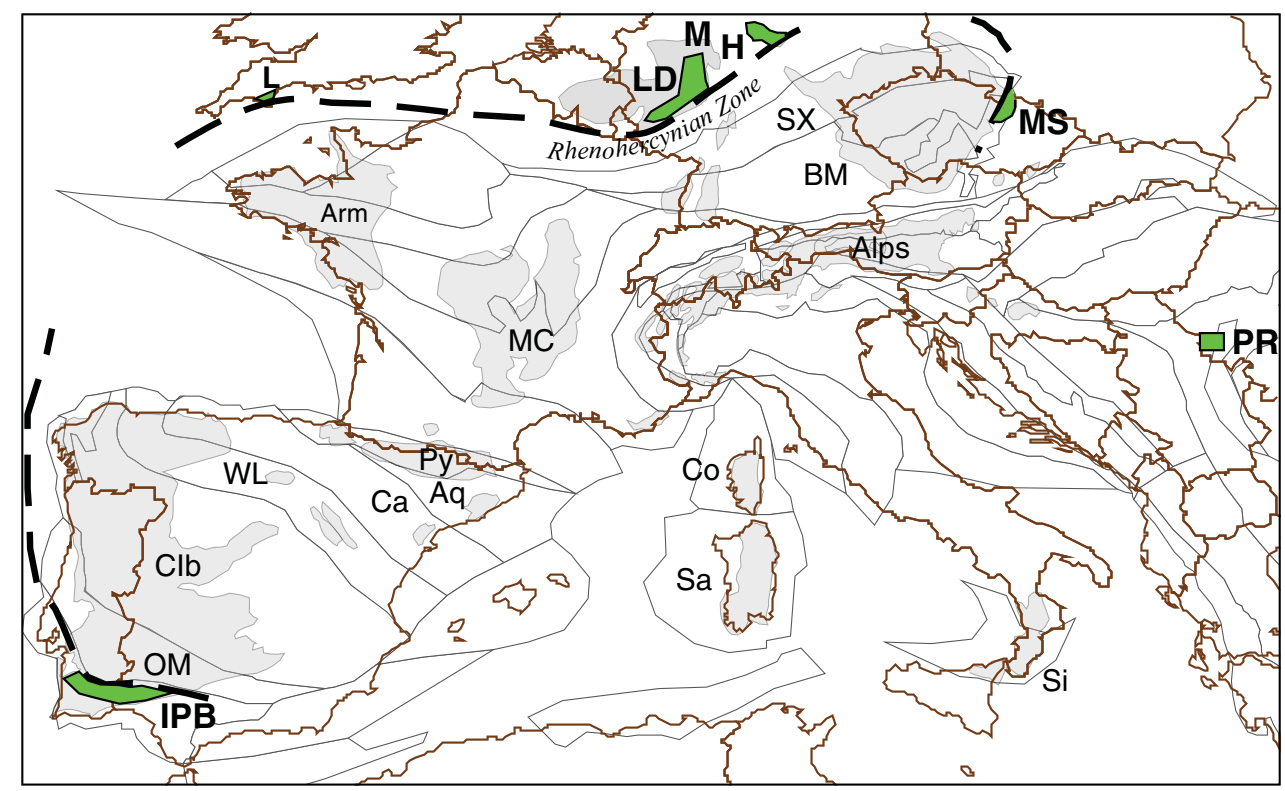

Fig. 1 Geographical distribution of Rhenohercynian ores sites (green) and their pre-Mesozoic basement hosts (light gray) in Central Europe (map modified after Stampfli et al. 2006). Black contours Geodynamic Units (GDUs, after Stampfli et al. 2006; Hochard 2008) inspired by Franke (1989). Brown contours Geographic borders. Dashed lines Contours of the Rhenohercynian Zone, after Ribeiro (1996). Ore sites: $H$ Harz area; IPB Iberian Pyrite Belt; $L D$ Lahn-Dill

belt consisting mainly of Devonian shales, graywackes and volcanics extends from the Rhine to the Harz mountains, through Czechoslovakia and the Carpathian mountains in Rumania," observing that "this belt appears to represent a metallotect for partly massive $\mathrm{Cu}-\mathrm{Pb}-\mathrm{Zn}-\mathrm{Fe}$ sulfide deposits with various amounts of barite" similar to the Iberian Pyrite Belt in southern Spain and Portugal.

Considering the advancements during the last decades in that field, the Late Variscan oroclinal bending, unfortunately, hinders comparing earlier structural elements (Figs. 1, 2). If plotted, instead, in a Devonian palinspastic reconstruction (Stampfli et al. 2013), the locations mentioned by Amstutz et al. (1971, e.g., Meggen pyrite-barite deposit; Rammelsberg mine, Harz; Iberian Pyrite Belt) would be explained best by the paleogeographic situation during the Devonian, where only the Carpathian localities mentioned by Kräutner (1970) would need a discussion (Fig. 3, see below).

We do not intend to rebuild the well-known geology of the Rhenohercynian domain (e.g., Franke 1995, 2000; Franke and Engel 1986; Oncken et al. 2000), being part of the Avalonian units of the Old Red continent, but we want to correlate the pre-orogenic to synorogenic sedimentation (Middle Devonian to Lower Carboniferous) in the Rhenohercynian Zone with the contemporaneous volcanic events (Nesbor et al. 1993; Königshof et al. 2010) and the related area; $L$ Lizard area; $M$ Meggen deposit; $M S$ Moravo-Silesian area; $P R$ Carpathian Pojana Rusca area. Geodynamic units: Arm Armorica; $A q$ Aquitaine; $B M$ Bohemian Massif + Black Forest-Vosges; $C I b$ Central Iberian basement, $\mathrm{Ca}$ Cantabrian terrane; $\mathrm{Co}$ Corsica; $\mathrm{MC}$ French Massif Central; OM Ossa Morena; Py Pyrenees; $S a$ Sardinia; Si Sicilia; Sx Saxothuringian Zone; WL West Asturian-Leonese Zone

ore sites (Fig. 2). Still highly debated, the subducting Rheic Ocean, the rifting/spreading of the Rhenohercynian Ocean and the Variscan orogenic events are supposed to play a major role in this scenario (compare Figs. 7, 8 in Stampfli et al. 2013). A reappraisal, including data on detrital zircons (Zeh and Gerdes 2010; Eckelmann et al. 2014b), as well as new data, allows presenting new ideas in connecting the north-directed subduction of the Rheic Ocean with the formation of ore deposits in the Rhenohercynian domain (Von Raumer et al. 2014).

\section{Stratigraphic considerations}

After Walliser's (1981) early section across the Rhenohercynian domain, Ziegler (1988, his Tab. 24) depicted a Silurian carbonate platform for the Rhenish Massif east of Rhine, at present interpreted as a nappe stack (Eckelmann et al. 2014a), thrust over the Devonian stratigraphic column including its rift-induced deposits, not testifying for any major orogenic event during this time period. Stets and Schäfer (2009) gave an impressive example for the Rhenish domains of the Lahn-Dill area to illustrate the Lower Devonian land-sea transitions at the northern margin of the Rhenohercynian Basin. Forming a large shelf of the Old Red continent (Doublier et al. 2012), its southernmost 


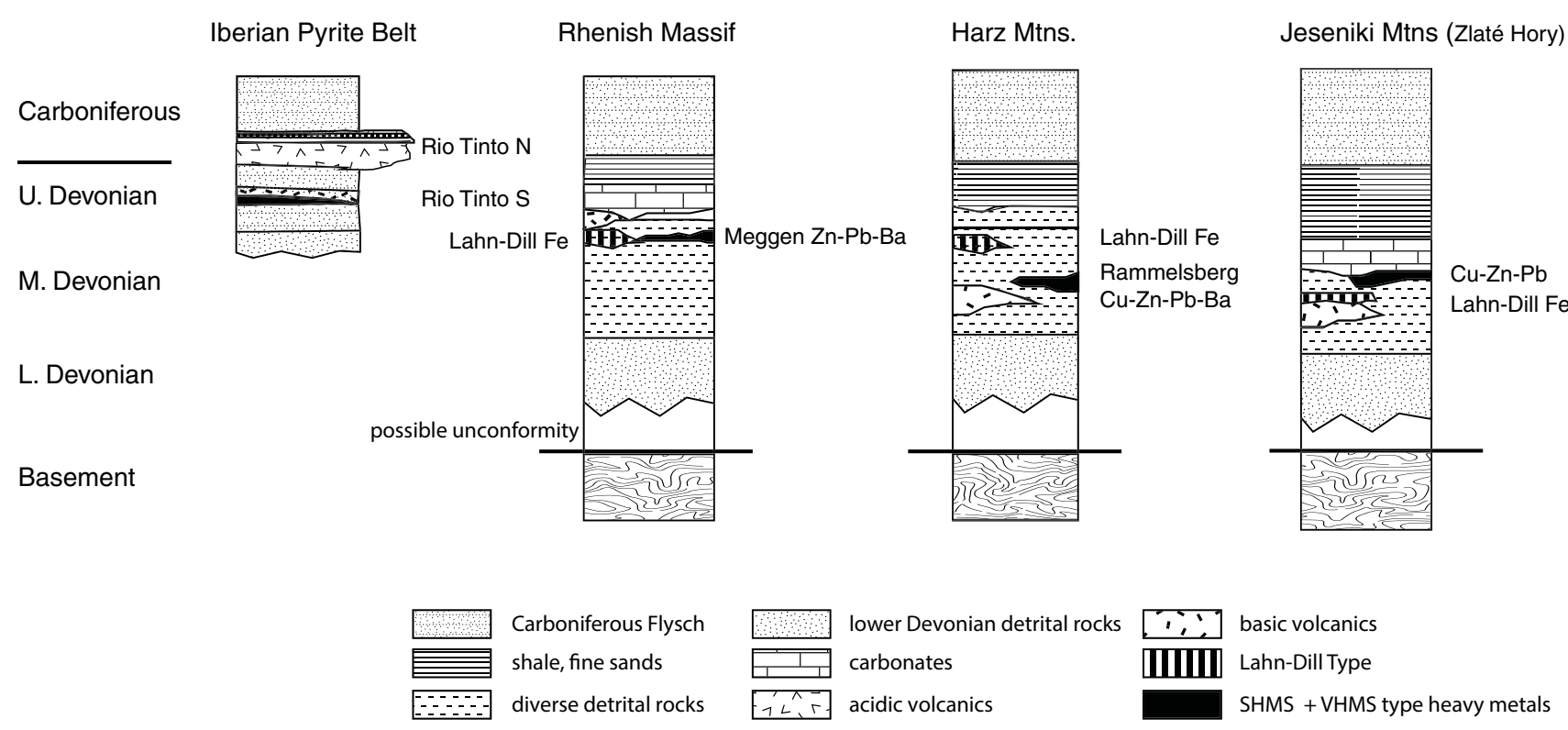

Fig. 2 Schematic lithostratigraphical comparison of Rhenohercynian ore sites (after Large 2003b; Tornos 2006), oversimplified lithostratigraphical sketches: with Carboniferous flysch deposits, and Lower Carboniferous sandy shale and acidic volcanics (Rio Tinto, northern Rio Tinto belt), and Upper to Lower Devonian dominant detrital rock series with intercalations of carbonates and metabasic volcanics, the Middle Devonian series hosting the base metal deposits-SHMS, VHMS and Lahn-Dill-type (Fe oxides) deposits (consult references for lithological details). From west to east: Iberian Pyrite Belt (after

margin was limited by a seismo-tectonic rise, furnishing clastic sedimentary input from the south during the Siegenian (Pragian, Stets and Schäfer 2009), thus leading to the conclusion that South Avalonia and North Avalonia, by then, were not yet separated. Contemporaneously, the north-subducting branch of the Rheic Ocean triggered the formation of a back-arc-type sedimentary trough within the southern border of the continental crust of Laurussia (Old Red continent).

The above-mentioned sedimentary input from the south would be represented by the "Taunus quartzite" (Stets and Schäfer 2009, 2011), its former source area being, supposedly, the northern part of the German Crystalline Rise (South Avalonia). During the Middle Devonian, when the Rhenohercynian Ocean began to open, an oceanic surface circulation pattern is observed not only in the Harz mountains (Oczlon 1992; Hüneke 2007, 2008), but also in the Moroccan eastern Meseta, most probably belonging to the Rhenohercynian domain (comp. Herbig and Aretz 2013), near the South Portuguese units (Fig. 3, eM). The formation of olistostromes (Salamon 2008) as well as contemporaneous bimodal volcanism (Nesbor 2004) plead for an active rifting period. Black shales were deposited in local troughs, and reefs formed along crests during times of reduced volcanic activity (Königshof et al. 2010).
Tornos 2006); Rhenish deposits, Harz domain and Moravo-Silesia (Izera) deposits (after Large 2003b). Two main events can be distinguished, first - a main Middle to Upper Devonian formation of SHMS, volcanogenic massive sulfide deposits (VHMS) and LahnDill type (Fe oxides) deposits, observed in all sections; second-main event of Tournaisian age VHMS, mainly observed in the northern Rio Tinto domain of the Iberian Pyrite Belt, but also expressed in the other domains

A comparable evolution should be expected for the South Iberian Pyrite Zone. Although strongly transformed during Late Variscan wrenching (Pereira et al. 2012), Late Devonian rifting of the Laurussian shelf was recognized in the South Portuguese Zone (Oliveira 1990; Silva et al. 1990). But the early findings of Lower Carboniferous trilobites in the South Portuguese Zone (Frischmuth 1968, Oliveira et al. 1979), proving the narrow relationship with contemporaneous endemic trilobites of the Harz area in the Rhenohercynian domain, did not yet enter as a valuable argument for paleogeographic considerations: The respective South Portuguese fossil sites should be located in a Devonian reconstruction of the Rhenohercynian Zone (comp. Fig. 3, PO) in the western prolongation of the Harz localities.

Dating of detrital zircons from the Rhenish Massif allowed for recognizing the original distribution of the strongly tectonized domains. Eckelmann et al. (2014a, b their Fig. 8) reconstructed from Devonian and Early Carboniferous siliciclastic rocks a plate-tectonic model which includes a northsubducting northern limit of the Rheic Ocean (subduction, rollback), constraining subduction-related volcanism from the Early Devonian to the Early Carboniferous and confirming the existence of the Rheic Ocean until the Early Carboniferous. In this context, the large similarity of the geochemical pattern of the Silurian rhyolites ("Sericit-Gneis," Meisl 1995) 


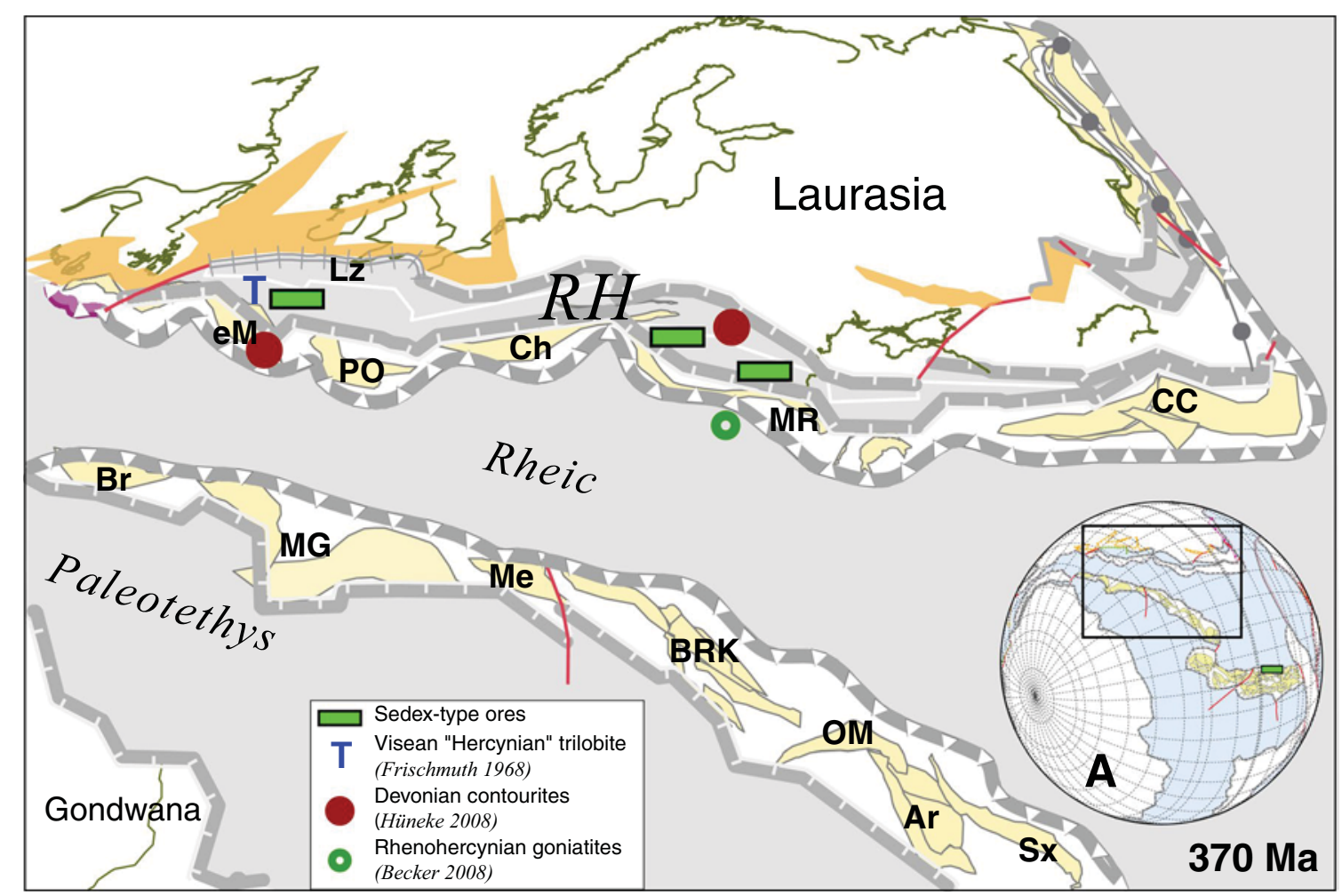

Fig. 3 Palinspastic map for the Rhenohercynian domain during the Middle Devonian (modified after Stampfli et al. 2011) and the corresponding Geodynamic Units (Hochard 2008), before oroclinal bending (Martínez Catalán 2012). In orange: continental rifts. Green rectangles represent from left to right (west to east): South Iberian domain (IPB in Fig. 1), near to EM and PO (see below); Rhenisch Lahn-Dill and Meggen, and Harz regions (LD, M, H, in Fig. 1); Moravo-Silesian domain (MS in Fig. 1). Inset A with limits of map area $B$ and supposed site of the Carpathian Pojana Rusca ore depos-

from the southern border of the Taunus (phyllite zone) and the rhyodacitic and rhyolitic rocks intercalated in the sedimentary succession of the Devonian and Lower Carboniferous has to be mentioned (Figs. 4, 5).

\section{Volcanic evolution}

Intensive volcanic activities accompanied the sedimentation on the southern shelf of Laurussia, where two fundamental different types of magmatic evolution characterize the plate-tectonic environment: continental intraplate and subduction-related volcanism.

\section{Intraplate volcanism}

Intraplate volcanism started during the Early Givetian on the southern shelf of Laurussia. Several thousand meters of clastic sediments, accumulating at the subsiding Devonian margin of the Old Red continent, formed the basis its (green rectangle). The Meguma terrane: Br New Brunswick; MG Meguma; Me Moroccan Meseta. The Armorica terrane: BRK BeticsRif-Kabbilies; OM Ossa Morena; Ar Armorica s.str; Sx Saxothuringia. Along the Eurasian margin, the opening of the Rhenohercynian Ocean (RH) has detached the Hanseatic terrane (former part of South Avalonia, compare Stampfli et al. 2011) from the mainland: eM eastern Meseta (comp. Herbig and Aretz 2013); Po South Portuguese Zone; Ch Channel; Lz Lizard; MR Mid-German Crystalline Rise; CC Caucasus

of the volcanic deposits. Two volcanic cycles can be distinguished: an alkaline Devonian and a tholeiitic Lower Carboniferous cycle (Nesbor 2004). The Devonian cycle comprised two phases, a bimodal main phase (Givetian-Frasnian phase) and a primitive basaltic late phase (Famennian phase). The main phase, lasting intermittently through Givetian and Early Frasnian time, was characterized by alkali basaltic to basanitic melts and their silicarich differentiates (Fig. 4). Huge submarine volcanic edifices were built, occasionally reaching the sea level. Times of less volcanic activity gave rise to the formation of iron ore deposits of the Lahn-Dill type and allowed reef development (Nesbor et al. 1993; Königshof et al. 2010). The corresponding bimodal trachytic to peralkaline rhyolitic volcanoes formed local islands (with plants and marginal reefs) in a shelf-sea of maximal $300 \mathrm{~m}$ depth (Fig. 7).

By the end of this bimodal volcanism, in the Early Frasnian, an intensive erosion of the numerous volcanoes is documented in the heterogeneous volcaniclastic sediments of the Dillenburg Formation. During the Late Famennian, 


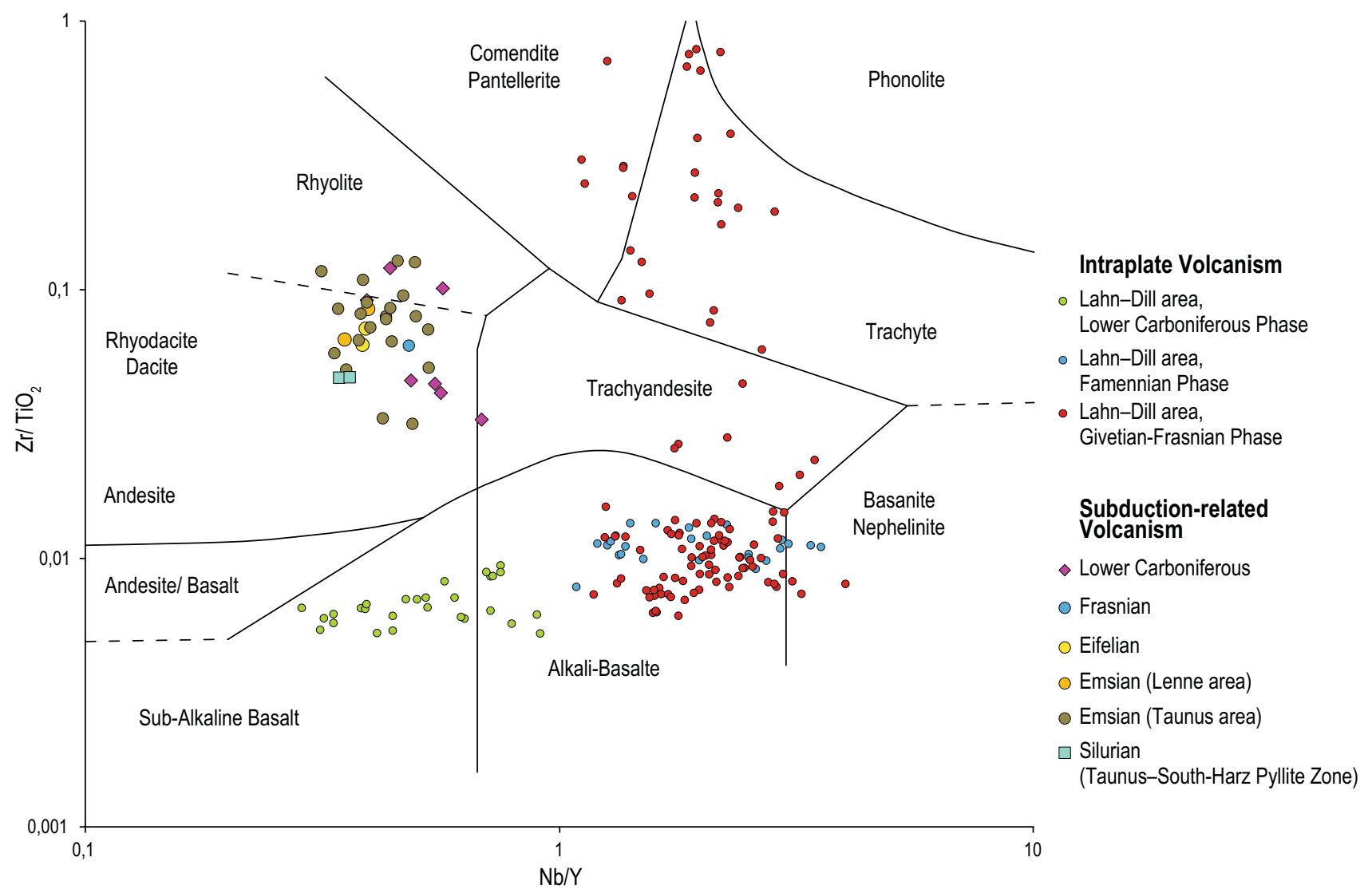

Fig. 4 Chemical discrimination of volcanic and volcaniclastic rocks of the Rheinisches Schiefergebirge in the $\mathrm{Zr} / \mathrm{TiO} \mathrm{O}_{2}$ versus $\mathrm{Nb} / \mathrm{Y}$ diagram of Winchester and Floyd (1977). Analyses of Lahn-Dill area from Nesbor 2004. (See Table 2 in electronic data-set)

Fig. 5 Trace element averages of Devonian and Lower Carboniferous dacitic/rhyodacitic and rhyolitic lapilli and ash tuffs/tuffites and metarhyolites in comparison with Silurian meta-rhyolites in the Mantle-normalized multivariation diagram of MacDonald and Sun (1995), indicating a typical trend of subduction-related character. Specific localities: comp. Table 1

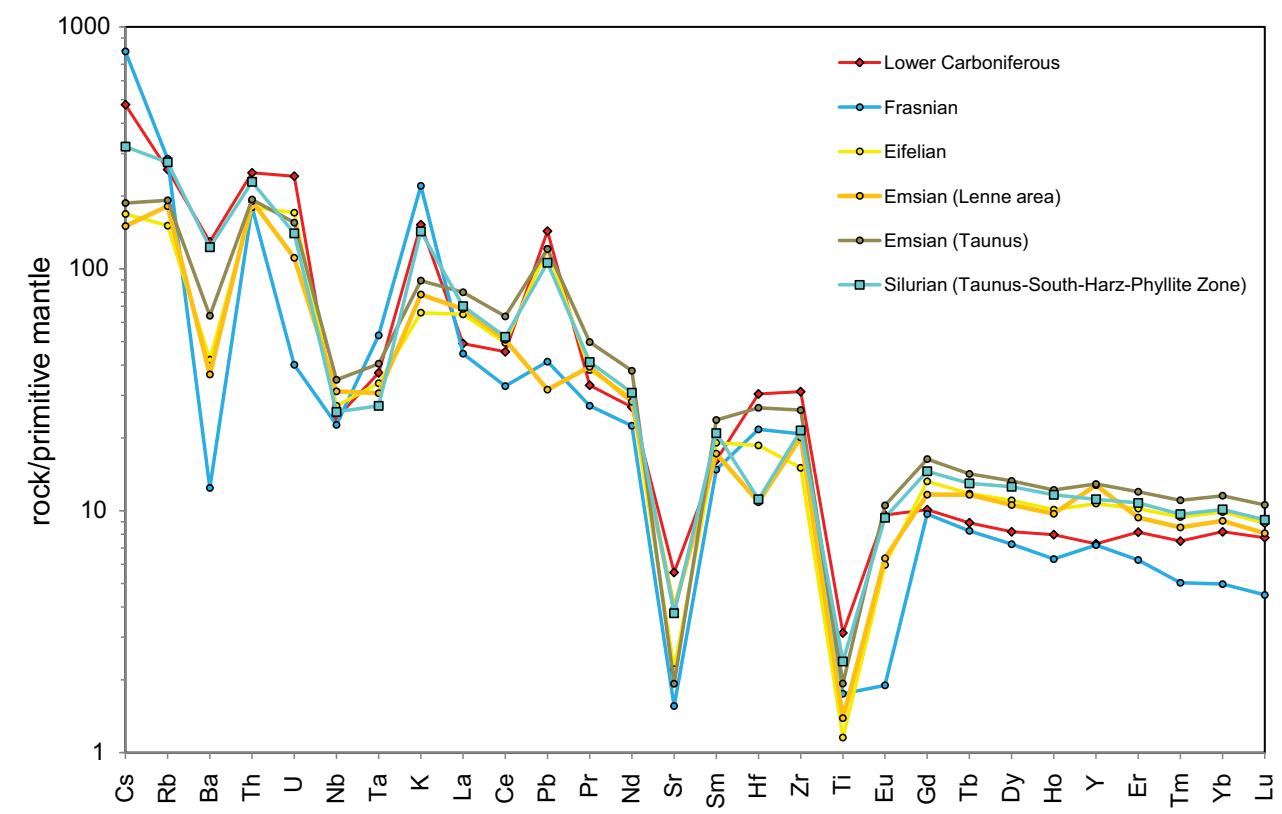

volcanic activity produced only minor quantities of primitive basaltic melts typical of late volcanic phases, carrying spinel-peridotitic mantle xenoliths.
Extension on the southern shelf of Laurussia continued during the Early Carboniferous and, combined with an increasing subsidence of the continental crust, was 
accompanied by a moderate increase in water depth. These processes are reflected by a change in the sedimentary environment as well as in the reviving volcanic activities. The melts produced were almost entirely tholeiitic now in contrast to the Devonian alkali basaltic to basanitic composition thereby still exhibiting intraplate affinities. Noticeable is the increase in aluminum in picotites enclosed in the olivine phenocrysts of the basalts, being an indication for ascending juvenile magma from deep mantle sources.

Large pillow volcanoes were created. Sills and dikes at subvolcanic levels are frequent as well. Volcanism ended with a primitive alkaline phase just prior to the onset of flysch sedimentation. The alkali basaltic melts of this phase transported numerous, partly very large spinel-lherzolitic mantle xenoliths from deep mantle sources (Nesbor 1997, 2004, 2007).

Sills and dikes of the Devonian as well as of the Carboniferous cycle are widely distributed in the entire Rheinisches Schiefergebirge (e.g., Taunus and Siegen anticline) and Harz proving the widespread extent of the volcanic activities, surpassing the region of the Lahn-Dill and Kellerwald areas.

\section{Subduction-related volcanism}

In contrast to the hitherto described intraplate volcanics, rhyodacitic to rhyolitic magmas represent typical products of subduction-related volcanism. This volcanic activity started in the Emsian stage of the Lower Devonian. Widespread ignimbrites and volcanic fall out deposits-often redeposited-are intercalated in the sedimentary succession between the Ebbe anticline in the northwest and the southern part of the Taunus at the southern margin of the Rhenish Massif. Several volcanic centers were located in the area of the northeastern part of the Siegen anticline where massive volcanic successions of dacitic/rhyodacitic and rhyolitic composition are exposed (Heyckendorf 1985). In the southeastern part of the Rheinisches Schiefergebirge, the source area of the mainly dacitic/rhyodacitic and rhyolitic pyroclastics, being frequently intercalated within the siliciclastic rock pile, is uncertain. Kirnbauer (1991) proposed a source area northeast, east or southeast of the Rheinisches Schiefergebirge with deposition of the pyroclastic material on the outer shelf area or on the slope of the southern margin of Laurussia. It is supposed that also the subduction-related volcanism is bimodal, but only the products of highly explosive plinian-type eruptions may have reached the Rhenohercynian domain, the more basic, less explosive members not attaining that range of distribution.

Due to just very low-grade metamorphic overprint, most of the volcanic products are well preserved, and the typical phenocrysts for rhyodacites and rhyolites, predominantly quartz, plagioclase and some alkali feldspar and biotite, can be identified. In the former glassy juvenile particles of the predominantly coarse-grained pyroclastic deposits, numerous vesicles are still visible, and Y-shaped former glass shards are a typical feature (Scherp 1983).

This type of volcanic activity continued through the Devonian right into the Visean. However, there is a decrease in grain size of the pyroclastic particles at the beginning of the Middle Devonian. From that time, numerous extremely fine-grained distal ash fall deposits, often hard to be detected, are intercalated within the thick pile of sedimentary rocks (Dehmer et al. 1989). From the Visean onwards, the grain size of the ash layers increased again (van Amerom et al. 2001).

Although more or less altered by diagenetic and/or hydrothermal processes, the volcanic rocks can still be classified by immobile elements. Plotting in the discrimination diagram in the fields of dacite, rhyodacite and rhyolite (Fig. 4), their typical calk-alkaline trend in the primitive mantle-normalized multivariant diagram (Fig. 5; Table 1) would support their subduction-related character (see discussion below).

\section{Short characteristics of the ore types}

Following Large (2003a, b), the ore deposits of the Rhenohercynian Zone comprise sediment-hosted massive sulfide deposits (SHMS, Rammelsberg, Meggen), volcanogenic massive sulfide deposits (VHMS) and Lahn-Dill-type (Fe oxides) deposits (Fig. 2), the latter to be distinguished from iron ore deposits ("Schalstein-Lager"), marking interruptions of volcanic activity and containing locally sulfidic ores. All being in some sense related to the platetectonic evolution of the Rhenohercynian Zone, they comprise, besides the Rhenish and the Harz occurrences, also domains from the Moravo-Silesian units in the southeastern part of the Bohemian Massif (Aichler et al. 1995). A comparable situation can be deduced for the Iberian Pyrite Belt (Tornos 2006), where basement with a Devonian phyllite-quartzite (PQ) sedimentary cover was crosscut by percolating hydrothermal fluids, resulting in massive sulfides formed by stratabound replacements and by brine pools. A subsequent Tournaisian volcano-sedimentary evolution, comprising the emplacement of felsic domes and volcaniclastic sediments, metasomatic and hydrothermal events resulted in the ores in the northern part of Rio Tinto, and comparable minor hydrothermal events are documented in the eastern prolongation of the Rhenohercynian Zone from the Harz area, and from the Rhenisch and Moravo-Sileasian domains (see below).

The Devonian post-rift thermal subsidence and extensional pulses triggered facies and thickness variations, the emplacement of mafic volcanics and the formation of 
Table 1 Geochemical composition of subduction- related volcanic and volcaniclastic rocks of the Rheinish Massif. Analysis G. Wörner, Univ. Göttingen

\begin{tabular}{|c|c|c|c|c|c|c|c|}
\hline Stratigraphic unit & 1 & 1 & 1 & 1 & 1 & 1 & 1 \\
\hline Sample & 26,897 & 29,255 & 29,399 & 34,670 & 46,312 & 46,313 & 46,314 \\
\hline GauB-Kriiger (R) & $3,494,790$ & $3,494,760$ & $3,494,790$ & $3,476,950$ & $3,464,460$ & $3,464,460$ & $3,464,460$ \\
\hline GauB-Kriiger (H) & $5,659,400$ & $5,659,400$ & $5,659,400$ & $5,675,410$ & $5,609,430$ & $5,609,430$ & $5,609,430$ \\
\hline Rock type & Dacitic ash tuff & Dacitic ash tuff & Dacitic ash tuff & Dacitic ash tuff & $\begin{array}{l}\text { Rtiyodacitic ash } \\
\text { tuff }\end{array}$ & $\begin{array}{l}\text { Rhyodacitic ash } \\
\text { tuff }\end{array}$ & $\begin{array}{l}\text { Rhyodacitic ash } \\
\text { tuff }\end{array}$ \\
\hline $\mathrm{SiO}_{2}(\%)$ & 41.00 & 43.87 & 43.46 & 48.76 & 63.78 & 72.33 & 67.66 \\
\hline $\mathrm{TiO}_{2}(\%)$ & 0.97 & 1.14 & 1.05 & 0.85 & 0.35 & 0.18 & 0.20 \\
\hline $\mathrm{Al}_{2} \mathrm{O} 3(\%)$ & 22.98 & 24.31 & 24.69 & 22.01 & 17.75 & 13.55 & 16.38 \\
\hline $\mathrm{Fe}_{2} \mathrm{O} 3 \mathrm{t}(\%)$ & 5.75 & 6.18 & 6.26 & 8.55 & 3.40 & 1.92 & 2.56 \\
\hline $\mathrm{MnO}(\%)$ & 0.16 & 0.14 & 0.10 & 0.21 & 0.07 & 0.08 & 0.11 \\
\hline $\mathrm{MgO}(\%)$ & 2.92 & 2.78 & 3.09 & 3.90 & 1.81 & 1.05 & 1.25 \\
\hline $\mathrm{CaO}(\%)$ & 6.74 & 4.54 & 3.59 & 1.11 & 0.73 & 1.09 & 0.74 \\
\hline $\mathrm{Na}_{2} \mathrm{O}(\%)$ & 0.18 & 1.51 & 0.17 & 2.69 & 1.50 & 0.69 & 2.47 \\
\hline $\mathrm{K}_{2} \mathrm{O}(\%)$ & 4.79 & 3.36 & 4.91 & 3.54 & 5.81 & 4.55 & 4.25 \\
\hline $\mathrm{P}_{2} \mathrm{O}_{5}(\%)$ & 0.33 & 0.39 & 0.34 & 0.37 & 0.10 & 0.04 & 0.06 \\
\hline L.O.I. (\%) & 12.97 & 10.99 & 10.70 & 8.14 & 4.22 & 3.65 & 3.43 \\
\hline $\mathrm{Ba}(\mathrm{ppm})$ & 678 & 393 & 690 & 1085 & 1339 & 950 & 666 \\
\hline $\mathrm{Co}(\mathrm{ppm})$ & 15 & 11 & 19 & 15 & 12 & 8 & 8 \\
\hline $\mathrm{Cr}(\mathrm{ppm})$ & 21 & 38 & 20 & 43 & 4 & 10 & 6 \\
\hline $\mathrm{Cu}(\mathrm{ppm})$ & 7 & 18 & 85 & 51 & 5 & 719 & 945 \\
\hline $\mathrm{Ga}(\mathrm{ppm})$ & 28 & 27 & 30 & 29 & 22 & 16 & 23 \\
\hline $\mathrm{Nb}(\mathrm{ppm})$ & 17 & 16 & 18 & 13 & 19 & 14 & 16 \\
\hline $\mathrm{Ni}(\mathrm{ppm})$ & 29 & 24 & 36 & 63 & 28 & 25 & 22 \\
\hline $\mathrm{Pb}(\mathrm{ppm})$ & 54 & 49 & 12 & 23 & 3 & 294 & 272 \\
\hline $\mathrm{Rb}(\mathrm{ppm})$ & 163 & 109 & 173 & 133 & 206 & 151 & 155 \\
\hline $\mathrm{Sc}(\mathrm{ppm})$ & 10 & 15 & 11 & 9 & 13 & 7 & 6 \\
\hline $\mathrm{Sr}(\mathrm{ppm})$ & 110 & 112 & 76 & 159 & 91 & 72 & 154 \\
\hline $\mathrm{V}(\mathrm{ppm})$ & 63 & 100 & 73 & 121 & 34 & 18 & 22 \\
\hline $\mathrm{Y}(\mathrm{ppm})$ & 35 & 29 & 33 & 19 & 47 & 25 & 36 \\
\hline $\mathrm{Zn}(\mathrm{ppm})$ & 58 & 157 & 51 & 159 & 51 & 75 & 55 \\
\hline $\mathrm{Zr}(\mathrm{ppm})$ & 444 & 471 & 468 & 280 & 316 & 179 & 243 \\
\hline Cs (ppm) & 6.19 & 11.01 & 10.38 & 13.72 & 10.05 & 8.81 & 16.64 \\
\hline $\mathrm{La}(\mathrm{ppm})$ & 25.50 & 10.37 & 26.26 & 44.02 & 23.26 & 13.46 & 31.03 \\
\hline $\mathrm{Ce}(\mathrm{ppm})$ & 47.86 & 18.79 & 63.23 & 86.26 & 59.15 & 33.91 & 70.56 \\
\hline $\operatorname{Pr}(p p m)$ & 7.46 & 3.65 & 8.26 & 9.81 & 6.99 & 3.98 & 8.01 \\
\hline $\mathrm{Nd}(\mathrm{ppm})$ & 28.87 & 15.50 & 32.23 & 34.48 & 27.13 & 15.26 & 29.14 \\
\hline $\mathrm{Sm}(\mathrm{ppm})$ & 5.33 & 3.26 & 5.73 & 5.40 & 5.84 & 2.93 & 5.59 \\
\hline $\mathrm{Eu}(\mathrm{ppm})$ & 1.36 & 0.90 & 1.33 & 1.16 & 1.16 & 0.57 & 0.94 \\
\hline Gd (ppm) & 4.77 & 3.20 & 4.81 & 4.27 & 5.87 & 3.09 & 4.96 \\
\hline $\mathrm{Tb}(\mathrm{ppm})$ & 0.73 & 0.50 & 0.73 & 0.53 & 1.11 & 0.58 & 0.87 \\
\hline Dy (ppm) & 4.36 & 3.16 & 4.41 & 2.93 & 8.37 & 4.38 & 6.28 \\
\hline Ho (ppm) & 0.86 & 0.65 & 0.89 & 0.56 & 1.93 & 1.07 & 1.47 \\
\hline $\operatorname{Er}(\mathrm{ppm})$ & 2.28 & 1.87 & 2.37 & 1.39 & 6.04 & 3.33 & 4.53 \\
\hline $\mathrm{Tm}(\mathrm{ppm})$ & 0.32 & 0.27 & 0.33 & 0.20 & 0.95 & 0.50 & 0.65 \\
\hline $\mathrm{Yb}(\mathrm{ppm})$ & 1.97 & 1.73 & 2.08 & 1.29 & 6.88 & 3.78 & 4.73 \\
\hline $\mathrm{Lu}(\mathrm{ppm})$ & 0.29 & 0.26 & 0.31 & 0.20 & 1.01 & 0.55 & 0.68 \\
\hline $\mathrm{Hf}$ (ppm) & 10.85 & 10.74 & 12.33 & 5.19 & 9.83 & 6.06 & 7.45 \\
\hline $\mathrm{Ta}(\mathrm{ppm})$ & 1.06 & 0.81 & 1.09 & 1.02 & 1.29 & 0.93 & 1.18 \\
\hline Th (ppm) & 18.55 & 11.10 & 25.83 & 34.34 & 21.90 & 17.35 & 22.97 \\
\hline
\end{tabular}


Table 1 continued

\begin{tabular}{|c|c|c|c|c|c|c|}
\hline $\mathrm{U}(\mathrm{ppm})$ & 1.68 & 0.69 & 4.43 & 7.10 & 6.13 & 6.17 \\
\hline Stratigraphic unit & & 2 & 3 & 3 & 4 & 4 \\
\hline Sample & & 45,302 & 45,223 & 46,290 & 46,396 & 46,397 \\
\hline GauB-Kriiger (R) & & $3,509,304$ & $3,501,306$ & $3,501,323$ & $3,438,520$ & $3,441,540$ \\
\hline GauB-Kriiger $(\mathrm{H})$ & & $5,661,296$ & $5,660,789$ & $5,660,833$ & $5,660,970$ & $5,663,195$ \\
\hline Rock type & & Rhyolitic ash tuff & Rhyolitic ash tuff & Rhyolitic ash tuff & Meta-rhyolite & Meta-rhyolite \\
\hline $\mathrm{SiO}_{2}(\%)$ & & 46.24 & 79.61 & 76.43 & 72.17 & 76.77 \\
\hline $\mathrm{TiO}_{2}(\%)$ & & 0.35 & 0.18 & 0.29 & 0.30 & 0.26 \\
\hline $\mathrm{Al}_{2} \mathrm{O} 3(\%)$ & & 22.31 & 8.24 & 12.53 & 13.18 & 12.57 \\
\hline $\mathrm{Fe}_{2} \mathrm{O} 3 \mathrm{t}(\%)$ & & 2.07 & 1.91 & 2.64 & 2.42 & 2.39 \\
\hline $\mathrm{MnO}(\%)$ & & 0.02 & 0.08 & 0.03 & 0.14 & 0.02 \\
\hline $\mathrm{MgO}(\%)$ & & 2.26 & 1.63 & 1.09 & 0.61 & 0.29 \\
\hline $\mathrm{CaO}(\%)$ & & 5.74 & 2.00 & 0.04 & 1.71 & 0.03 \\
\hline $\mathrm{Na}_{2} \mathrm{O}(\%)$ & & 0.04 & 0.25 & 2.13 & 2.06 & 3.99 \\
\hline $\mathrm{K}_{2} \mathrm{O}(\%)$ & & 6.37 & 1.42 & 2.39 & 2.89 & 1.64 \\
\hline $\mathrm{P}_{2} \mathrm{O}_{5}(\%)$ & & 0.12 & 0.04 & 0.04 & 0.06 & 0.07 \\
\hline L.O.I. (\%) & & 13.67 & 4.03 & 1.92 & 4.34 & 2.01 \\
\hline $\mathrm{Ba}(\mathrm{ppm})$ & & 82 & 222 & 334 & 288 & 195 \\
\hline Co (ppm) & & 7 & 3 & 4 & 2 & 2 \\
\hline $\mathrm{Cr}(\mathrm{ppm})$ & & 11 & 12 & 34 & 8 & 12 \\
\hline $\mathrm{Cu}(\mathrm{ppm})$ & & 118 & 6 & 1 & 1 & 3 \\
\hline $\mathrm{Ga}(\mathrm{ppm})$ & & 24 & 11 & 18 & 18 & 16 \\
\hline $\mathrm{Nb}(\mathrm{ppm})$ & & 15 & 13 & 23 & 22 & 19 \\
\hline Ni (ppm) & & 32 & 16 & 19 & 5 & 5 \\
\hline $\mathrm{Pb}(\mathrm{ppm})$ & & 6 & 23 & 13 & 3 & 7 \\
\hline $\mathrm{Rb}(\mathrm{ppm})$ & & 171 & 67 & 114 & 129 & 89 \\
\hline $\mathrm{Sc}(\mathrm{ppm})$ & & 7 & 7 & 7 & 9 & 8 \\
\hline $\mathrm{Sr}(\mathrm{ppm})$ & & 31 & 47 & 41 & 63 & 93 \\
\hline $\mathrm{V}(\mathrm{ppm})$ & & 35 & 18 & 27 & 15 & 21 \\
\hline $\mathrm{Y}(\mathrm{ppm})$ & & 31 & 33 & 59 & 56 & 54 \\
\hline $\mathrm{Zn}(\mathrm{ppm})$ & & 23 & 69 & 59 & 18 & 9 \\
\hline $\mathrm{Zr}(\mathrm{ppm})$ & & 218 & 111 & 205 & 251 & 171 \\
\hline Cs (ppm) & & 3.03 & 4.05 & 5.11 & 1.20 & 5.06 \\
\hline $\mathrm{La}(\mathrm{ppm})$ & & 28.91 & 30.61 & 53.40 & 63.80 & 24.70 \\
\hline $\mathrm{Ce}(\mathrm{ppm})$ & & 54.84 & 54.61 & 111.00 & 122.00 & 48.60 \\
\hline $\operatorname{Pr}(\mathrm{ppm})$ & & 6.90 & 6.40 & 13.00 & 14.30 & 5.69 \\
\hline $\mathrm{Nd}(\mathrm{ppm})$ & & 28.07 & 24.46 & 48.70 & 50.70 & 19.90 \\
\hline $\mathrm{Sm}(\mathrm{ppm})$ & & 6.01 & 5.32 & 10.20 & 10.00 & 3.99 \\
\hline $\mathrm{Eu}(\mathrm{ppm})$ & & 0.29 & 0.51 & 1.33 & 1.56 & 0.40 \\
\hline Gd (ppm) & & 5.27 & 4.90 & 9.49 & 9.11 & 3.57 \\
\hline $\mathrm{Tb}(\mathrm{ppm})$ & & 0.82 & 0.80 & 1.53 & 1.63 & 0.68 \\
\hline Dy (ppm) & & 4.90 & 5.15 & 9.72 & 9.87 & 4.37 \\
\hline Ho (ppm) & & 0.94 & 1.04 & 1.97 & 1.98 & 0.92 \\
\hline $\operatorname{Er}(\mathrm{ppm})$ & & 2.74 & 3.25 & 5.69 & 5.55 & 2.66 \\
\hline $\mathrm{Tm}$ (ppm) & & 0.34 & 0.46 & 0.82 & 0.77 & 0.39 \\
\hline $\mathrm{Yb}(\mathrm{ppm})$ & & 2.19 & 3.16 & 5.55 & 5.38 & 2.63 \\
\hline $\mathrm{Lu}(\mathrm{ppm})$ & & 0.30 & 0.44 & 0.76 & 0.73 & 0.36 \\
\hline Hf (ppm) & & 6.14 & 3.37 & 7.17 & 3.71 & 2.43 \\
\hline $\mathrm{Ta}(\mathrm{ppm})$ & & 1.96 & 0.90 & 1.59 & 1.50 & 0.76 \\
\hline Th (ppm) & & 14.30 & 11.89 & 16.70 & 19.40 & 10.90 \\
\hline
\end{tabular}


Table 1 continued

\begin{tabular}{|c|c|c|c|c|c|c|c|c|c|c|c|}
\hline \multicolumn{2}{|l|}{$\mathrm{U}(\mathrm{ppm})$} & \multicolumn{2}{|l|}{0.81} & \multicolumn{2}{|l|}{3.09} & \multicolumn{2}{|c|}{3.84} & \multicolumn{2}{|r|}{2.14} & \multicolumn{2}{|c|}{2.37} \\
\hline $\begin{array}{l}\text { Strati- } \\
\text { graphic } \\
\text { unit }\end{array}$ & 5 & 5 & 5 & 5 & 5 & 5 & 5 & 5 & 5 & 5 & 5 \\
\hline Sample & 46,257 & 46,259 & 46,260 & 46,262 & 46,263 & 46,264 & 46,265 & 46,266 & 46,268 & 46,269 & 46,270 \\
\hline $\begin{array}{l}\text { GauB- } \\
\text { Kriiger } \\
(\mathrm{R})\end{array}$ & $3,446,116$ & $3,450,197$ & $3,452,408$ & $3,451,683$ & $3,456,766$ & $3,459,753$ & $3,458,350$ & $3,460,068$ & $3,459,953$ & $3,459,953$ & $3,462,842$ \\
\hline $\begin{array}{l}\text { GauB- } \\
\text { Kriiger } \\
(\mathrm{H})\end{array}$ & $5,562,784$ & $5,563,663$ & $5,566,869$ & $5,572,436$ & $5,573,644$ & $5,574,780$ & $5,567,881$ & $5,569,251$ & $5,569,645$ & $5,569,645$ & $5,568,660$ \\
\hline Rock type & $\begin{array}{l}\text { Rhyolitic } \\
\text { ash tuff }\end{array}$ & $\begin{array}{l}\text { Rhyolitic } \\
\text { ash tuff }\end{array}$ & $\begin{array}{l}\text { Rhyolitic } \\
\text { ash tuff }\end{array}$ & $\begin{array}{l}\text { Rhyolitic } \\
\text { ash tuff }\end{array}$ & $\begin{array}{l}\text { Rhyolitic } \\
\text { ash tuff }\end{array}$ & $\begin{array}{r}\text { Rhyolitic } \\
\text { ash tuff }\end{array}$ & $\begin{array}{r}\text { Rhyolitic } \\
\text { ash tuff }\end{array}$ & $\begin{array}{r}\text { Rhyolitic } \\
\text { ash tuff }\end{array}$ & $\begin{array}{c}\text { Rhyolitic } \\
\text { ash tuff }\end{array}$ & $\begin{array}{l}\text { Rhyolitic } \\
\text { ash tuff }\end{array}$ & $\begin{array}{r}\text { Rhyolitic } \\
\text { ash tuff }\end{array}$ \\
\hline $\mathrm{SiO}_{2}(\%)$ & 71.64 & 75.16 & 73.32 & 73.84 & 71.62 & 75.36 & 77.95 & 74.69 & 81.76 & 65.64 & 75.54 \\
\hline $\mathrm{TiO}_{2}(\%)$ & 0.57 & 0.39 & 0.50 & 0.41 & 0.53 & 0.40 & 0.25 & 0.37 & 0.49 & 0.91 & 0.30 \\
\hline $\begin{array}{c}\mathrm{Al}_{2} \mathrm{O} 3 \\
(\%)\end{array}$ & 12.61 & 12.92 & 13.84 & 13.45 & 14.09 & 13.12 & 11.63 & 13.03 & 7.58 & 16.13 & 13.17 \\
\hline $\begin{array}{c}\mathrm{Fe}_{2} \mathrm{O} 3 \mathrm{t} \\
(\%)\end{array}$ & 3.93 & 3.15 & 3.71 & 3.38 & 3.93 & 2.82 & 2.58 & 3.18 & 4.27 & 6.17 & 2.56 \\
\hline $\mathrm{MnO}(\%)$ & 0.10 & 0.04 & 0.06 & 0.07 & 0.06 & 0.04 & 0.11 & 0.06 & 0.07 & 0.07 & 0.05 \\
\hline $\mathrm{MgO}(\%)$ & 1.30 & 1.18 & 0.97 & 1.24 & 1.71 & 1.00 & 0.98 & 1.32 & 1.08 & 2.06 & 1.07 \\
\hline $\mathrm{CaO}(\%)$ & 1.47 & 0.04 & 0.05 & 0.09 & 0.09 & 0.06 & 0.01 & 0.07 & 0.15 & 0.18 & 0.03 \\
\hline $\mathrm{Na}_{2} \mathrm{O}(\%)$ & 1.71 & 2.33 & 0.87 & 0.86 & 1.05 & 1.20 & 1.68 & 1.67 & 1.89 & 1.60 & 1.32 \\
\hline $\mathrm{K}_{2} \mathrm{O}(\%)$ & 2.46 & 2.29 & 3.28 & 3.46 & 3.15 & 3.02 & 2.29 & 2.74 & 0.52 & 3.09 & 2.84 \\
\hline $\mathrm{P}_{2} \mathrm{O}_{5}(\%)$ & 0.08 & 0.05 & 0.07 & 0.05 & 0.09 & 0.04 & 0.03 & 0.05 & 0.11 & 0.19 & 0.04 \\
\hline L.O.I. (\%) & 3.51 & 2.16 & 2.84 & 2.65 & 2.95 & 2.38 & 2.12 & 2.39 & 1.48 & 3.48 & 2.37 \\
\hline $\mathrm{Ba}(\mathrm{ppm})$ & 432 & 390 & 469 & 390 & 358 & 881 & 295 & 380 & 73 & 403 & 425 \\
\hline $\mathrm{Co}(\mathrm{ppm})$ & 9 & 5 & 10 & 6 & 8 & 4 & 4 & 7 & 10 & 14 & 4 \\
\hline $\mathrm{Cr}(\mathrm{ppm})$ & 92 & 45 & 69 & 51 & 90 & 39 & 28 & 53 & 131 & 143 & 38 \\
\hline $\mathrm{Cu}(\mathrm{ppm})$ & 3 & 0 & 7 & 1 & 3 & 1 & 1 & 1 & 1 & 10 & 1 \\
\hline $\mathrm{Ga}(\mathrm{ppm})$ & 18 & 20 & 20 & 21 & 20 & 21 & 16 & 18 & 9 & 20 & 19 \\
\hline $\mathrm{Nb}(\mathrm{ppm})$ & 23 & 27 & 28 & 30 & 23 & 33 & 22 & 23 & 12 & 20 & 23 \\
\hline $\mathrm{Ni}(\mathrm{ppm})$ & 38 & 28 & 41 & 39 & 37 & 18 & 24 & 30 & 37 & 66 & 24 \\
\hline $\mathrm{Pb}(\mathrm{ppm})$ & 21 & 19 & 30 & 20 & 18 & 16 & 21 & 22 & 9 & 16 & 22 \\
\hline $\mathrm{Rb}(\mathrm{ppm})$ & 117 & 108 & 144 & 153 & 144 & 133 & 106 & 136 & 24 & 140 & 131 \\
\hline $\mathrm{Sc}(\mathrm{ppm})$ & 11 & 10 & 10 & 8 & 15 & 12 & 5 & 7 & 2 & 14 & 7 \\
\hline $\mathrm{Sr}(\mathrm{ppm})$ & 86 & 44 & 39 & 28 & 32 & 29 & 33 & 39 & 43 & 51 & 34 \\
\hline $\mathrm{V}(\mathrm{ppm})$ & 57 & 35 & 51 & 34 & 60 & 26 & 23 & 40 & 51 & 113 & 31 \\
\hline $\mathrm{Y}(\mathrm{ppm})$ & 53 & 53 & 74 & 77 & 43 & 106 & 65 & 69 & 28 & 41 & 58 \\
\hline $\mathrm{Zn}(\mathrm{ppm})$ & 86 & 77 & 91 & 74 & 71 & 89 & 80 & 67 & 53 & 83 & 71 \\
\hline $\mathrm{Zr}(\mathrm{ppm})$ & 364 & 309 & 325 & 364 & 273 & 464 & 214 & 217 & 391 & 289 & 218 \\
\hline Cs (ppm) & 3.70 & 5.61 & 4.73 & 5.51 & 4.14 & 4.06 & 4.92 & 0.97 & 5.70 & 5.10 & 2.35 \\
\hline $\mathrm{La}(\mathrm{ppm})$ & 55.50 & 59.60 & 61.70 & 71.50 & 53.90 & 67.00 & 54.20 & 53.60 & 26.40 & 45.30 & 58.40 \\
\hline $\mathrm{Ce}(\mathrm{ppm})$ & 112.00 & 122.00 & 126.00 & 138.00 & 110.00 & 136.00 & 105.00 & 109.00 & 55.00 & 92.90 & 121.00 \\
\hline $\operatorname{Pr}(\mathrm{ppm})$ & 13.50 & 14.60 & 15.20 & 17.20 & 12.90 & 16.60 & 13.10 & 13.00 & 6.70 & 11.00 & 14.10 \\
\hline $\mathrm{Nd}(\mathrm{ppm})$ & 50.80 & 55.20 & 57.10 & 64.30 & 48.00 & 63.70 & 49.80 & 48.20 & 25.70 & 41.60 & 52.10 \\
\hline $\mathrm{Sm}(\mathrm{ppm})$ & 10.10 & 11.30 & 12.00 & 13.10 & 9.44 & 13.70 & 10.40 & 9.81 & 5.54 & 8.44 & 10.50 \\
\hline $\mathrm{Eu}(\mathrm{ppm})$ & 1.87 & 2.03 & 2.10 & 2.41 & 1.55 & 2.67 & 1.72 & 1.57 & 1.08 & 1.60 & 1.55 \\
\hline $\mathrm{Gd}(\mathrm{ppm})$ & 9.23 & 10.10 & 11.20 & 12.10 & 8.11 & 14.50 & 10.20 & 9.53 & 5.13 & 7.26 & 9.47 \\
\hline $\mathrm{Tb}(\mathrm{ppm})$ & 1.41 & 1.56 & 1.83 & 2.01 & 1.22 & 2.46 & 1.67 & 1.60 & 0.74 & 1.09 & 1.48 \\
\hline Dy (ppm) & 8.87 & 9.71 & 11.80 & 13.10 & 7.46 & 16.10 & 10.40 & 10.50 & 4.48 & 6.70 & 9.22 \\
\hline
\end{tabular}


Table 1 continued

\begin{tabular}{|c|c|c|c|c|c|c|c|c|c|c|c|c|}
\hline Ho (ppm) & 1.78 & 1.95 & 2.41 & 2.69 & 1.50 & 3.25 & 2.12 & 2.12 & 0.8 & 88 & 1.31 & 1.85 \\
\hline $\operatorname{Er}(\mathrm{ppm})$ & 5.13 & 5.67 & 6.92 & 7.74 & 4.32 & 9.23 & 5.96 & 6.0 & 2. & 47 & 3.72 & 5.33 \\
\hline $\operatorname{Tm}(\mathrm{ppm})$ & 0.72 & 0.82 & 1.00 & 1.10 & 0.62 & 1.30 & 0.86 & 0.8 & $0 . ?$ & 35 & 0.53 & 0.74 \\
\hline $\mathrm{Yb}(\mathrm{ppm})$ & 4.95 & 5.61 & 6.63 & 7.46 & 4.24 & 8.36 & 5.61 & 5.70 & 2.3 & 34 & 3.54 & 5.06 \\
\hline $\mathrm{Lu}(\mathrm{ppm})$ & 0.70 & 0.79 & 0.95 & 1.05 & 0.60 & 1.17 & 0.79 & 0.8 & $0 . ?$ & 34 & 0.50 & 0.69 \\
\hline $\mathrm{Hf}$ (ppm) & 8.55 & 8.85 & 8.66 & 10.30 & 7.37 & 9.28 & 6.89 & 7.27 & 6. & 71 & 7.10 & 7.81 \\
\hline $\mathrm{Ta}(\mathrm{ppm})$ & 1.47 & 1.75 & 1.68 & 1.93 & 1.54 & 1.80 & 1.47 & 1.5 & 0. & 69 & 1.27 & 1.68 \\
\hline Th (ppm) & 15.50 & 17.50 & 17.00 & 19.30 & 16.10 & 17.00 & 15.00 & 16.2 & 20 & 78 & 13.50 & 17.80 \\
\hline $\mathrm{U}(\mathrm{ppm})$ & 3.26 & 3.33 & 3.61 & 3.33 & 3.28 & 3.82 & 2.81 & 3.4 & 1. & 74 & 2.99 & 3.84 \\
\hline $\begin{array}{l}\text { Strati- } \\
\text { graphic } \\
\text { unit }\end{array}$ & 5 & 5 & 5 & 5 & 5 & 5 & 5 & 5 & 5 & 5 & 6 & 6 \\
\hline Sample & 46,271 & 46,273 & 46,274 & 46,275 & 46,276 & 46,277 & 46,278 & 46,280 & 46,281 & 46,282 & 45,357 & 45,358 \\
\hline $\begin{array}{l}\text { GauB- } \\
\text { Kriiger } \\
(\mathrm{R})\end{array}$ & $3,459,753$ & $3,463,136$ & $3,473,415$ & $3,472,690$ & $3,472,785$ & $3,467,869$ & $3,470,011$ & $3,471,581$ & $3,471,581$ & $3,471,700$ & $3,464,156$ & $3,457,558$ \\
\hline $\begin{array}{l}\text { GauB- } \\
\text { Kriiger } \\
(\mathrm{H})\end{array}$ & $5,574,780$ & $5,579,595$ & $5,585,966$ & $5,582,487$ & $5,582,378$ & $5,585,584$ & $5,580,085$ & $5,578,340$ & $5,578,340$ & $5,578,513$ & $35,559,861$ & $5,557,741$ \\
\hline $\begin{array}{l}\text { Rock } \\
\text { type }\end{array}$ & $\begin{array}{l}\text { Rhyolitic } \\
\text { ash } \\
\text { tuff }\end{array}$ & $\begin{array}{l}\text { Rhyolitic } \\
\text { ash } \\
\text { tuff }\end{array}$ & $\begin{array}{l}\text { Rhyolitic } \\
\text { ash } \\
\text { tuff }\end{array}$ & $\begin{array}{l}\text { Rhyolitic } \\
\text { ash } \\
\text { tuff }\end{array}$ & $\begin{array}{l}\text { Rhyolitic } \\
\text { ash } \\
\text { tuff }\end{array}$ & $\begin{array}{l}\text { Rhyolitic } \\
\text { ash } \\
\text { tuff }\end{array}$ & $\begin{array}{l}\text { Rhyolitic } \\
\text { ash } \\
\text { tuff }\end{array}$ & $\begin{array}{l}\text { Rhyolitic } \\
\text { ash } \\
\text { tuff }\end{array}$ & $\begin{array}{l}\text { Rhyolitic } \\
\text { ash } \\
\text { tuff }\end{array}$ & $\begin{array}{l}\text { Rhyolitic } \\
\text { ash } \\
\text { tuff }\end{array}$ & $\begin{array}{c}\text { Meta- } \\
\text { rhyo- } \\
\text { lite }\end{array}$ & $\begin{array}{l}\text { Meta- } \\
\text { rhyolite }\end{array}$ \\
\hline $\mathrm{SiO}_{2}(\%)$ & 74.78 & 79.29 & 76.37 & 81.55 & 78.68 & 80.17 & 75.86 & 79.91 & 78.37 & 73.84 & 70.64 & 70.63 \\
\hline $\mathrm{TiO}_{2}(\%)$ & 0.54 & 0.21 & 0.30 & 0.15 & 0.25 & 0.23 & 0.31 & 0.29 & 0.30 & 0.45 & 0.49 & 0.47 \\
\hline $\begin{array}{c}\mathrm{Al}_{2} \mathrm{O} 3 \\
(\%)\end{array}$ & 10.19 & 11.76 & 12.15 & 9.73 & 10.96 & 11.31 & 12.59 & 10.69 & 11.93 & 12.52 & 14.18 & 13.88 \\
\hline $\begin{array}{c}\mathrm{Fe}_{2} \mathrm{O} 3 \mathrm{t} \\
(\%)\end{array}$ & 4.35 & 2.05 & 2.65 & 2.17 & 2.46 & 1.81 & 2.81 & 2.09 & 2.05 & 4.37 & 3.37 & 3.38 \\
\hline $\begin{array}{r}\mathrm{MnO} \\
(\%)\end{array}$ & 0.11 & 0.04 & 0.07 & 0.03 & 0.05 & 0.06 & 0.03 & 0.12 & 0.06 & 0.05 & 0.05 & 0.06 \\
\hline $\begin{array}{r}\mathrm{MgO} \\
(\%)\end{array}$ & 1.14 & 0.86 & 1.23 & 1.23 & 1.30 & 0.79 & 1.58 & 0.63 & 0.80 & 1.64 & 0.76 & 0.60 \\
\hline $\mathrm{CaO}(\%)$ & 1.60 & 0.01 & 0.41 & 0.02 & 0.27 & 0.02 & 0.03 & 0.04 & 0.02 & 0.08 & 0.65 & 1.15 \\
\hline $\begin{array}{r}\mathrm{Na}_{2} \mathrm{O} \\
(\%)\end{array}$ & 1.56 & 0.36 & 1.00 & 0.08 & 0.69 & 0.34 & 0.57 & 0.78 & 0.25 & 0.75 & 3.56 & 3.21 \\
\hline $\mathrm{K}_{2} \mathrm{O}(\%)$ & 1.71 & 2.56 & 2.64 & 2.27 & 2.19 & 2.55 & 3.16 & 2.45 & 3.03 & 2.58 & 3.85 & 4.41 \\
\hline $\mathrm{P}_{2} \mathrm{O}_{5}(\%)$ & 0.09 & 0.02 & 0.04 & 0.02 & 0.03 & 0.03 & 0.03 & 0.05 & 0.04 & 0.08 & 0.14 & 0.13 \\
\hline $\begin{array}{l}\text { L.O.I. } \\
(\%)\end{array}$ & 3.31 & 2.31 & 2.67 & 2.24 & 2.49 & 2.37 & 2.78 & 2.17 & 2.56 & 2.91 & 1.86 & 1.47 \\
\hline $\mathrm{Ba}(\mathrm{ppm})$ & 483 & 506 & 568 & 382 & 463 & 345 & 598 & 310 & 386 & 332 & 815 & 807 \\
\hline $\begin{array}{l}\text { Co } \\
\qquad(\mathrm{ppm})\end{array}$ & 12 & 2 & 5 & 2 & 4 & 2 & 4 & 5 & 4 & 8 & 7 & 7 \\
\hline $\mathrm{Cr}(\mathrm{ppm})$ & 85 & 15 & 44 & 12 & 32 & 19 & 31 & 30 & 38 & 68 & 14 & 15 \\
\hline $\begin{array}{l}\mathrm{Cu} \\
\quad(\mathrm{ppm})\end{array}$ & 7 & 0 & 1 & 1 & 1 & 1 & 1 & 1 & 1 & 3 & 17 & 18 \\
\hline $\begin{array}{l}\mathrm{Ga} \\
\quad(\mathrm{ppm})\end{array}$ & 13 & 19 & 17 & 14 & 16 & 19 & 20 & 16 & 18 & 18 & 17 & 16 \\
\hline $\begin{array}{l}\mathrm{Nb} \\
\quad(\mathrm{ppm})\end{array}$ & 12 & 27 & 22 & 18 & 20 & 25 & 28 & 21 & 22 & 21 & 17 & 17 \\
\hline $\mathrm{Ni}(\mathrm{ppm})$ & 49 & 13 & 21 & 7 & 25 & 9 & 16 & 15 & 17 & 34 & 7 & 7 \\
\hline $\mathrm{Pb}(\mathrm{ppm})$ & 16 & 14 & 19 & 13 & 16 & 28 & 25 & 17 & 4 & 15 & 14 & 18 \\
\hline $\begin{array}{l}\mathrm{Rb} \\
\quad(\mathrm{ppm})\end{array}$ & 74 & 113 & 114 & 97 & 92 & 94 & 142 & 108 & 134 & 115 & 156 & 175 \\
\hline
\end{tabular}


Table 1 continued

\begin{tabular}{|c|c|c|c|c|c|c|c|c|c|c|c|c|}
\hline $\mathrm{Sc}(\mathrm{ppm})$ & 11 & 6 & 6 & 4 & 6 & 7 & 6 & 12 & 12 & 15 & 8 & 9 \\
\hline $\mathrm{Sr}(\mathrm{ppm})$ & 42 & 61 & 38 & 18 & 42 & 46 & 24 & 25 & 21 & 30 & 64 & 86 \\
\hline $\mathrm{V}(\mathrm{ppm})$ & 64 & 13 & 28 & 11 & 23 & 15 & 23 & 24 & 28 & 50 & 43 & 39 \\
\hline $\mathrm{Y}(\mathrm{ppm})$ & 28 & 60 & 51 & 47 & 52 & 51 & 60 & 49 & 41 & 58 & 50 & 46 \\
\hline $\mathrm{Zn}(\mathrm{ppm})$ & 54 & 64 & 68 & 53 & 68 & 70 & 75 & 25 & 25 & 83 & 51 & 46 \\
\hline $\mathrm{Zr}(\mathrm{ppm})$ & 179 & 269 & 234 & 160 & 201 & 291 & 293 & 247 & 214 & 229 & 231 & 220 \\
\hline Cs (ppm) & 2.68 & 3.96 & 3.02 & 3.48 & 2.30 & 4.76 & 3.27 & 3.72 & 3.49 & 6.25 & 7.20 & \\
\hline La (ppm) & 27.10 & 54.20 & 53.70 & 41.70 & 50.00 & 45.10 & 63.00 & 46.70 & 51.30 & 48.80 & 46.55 & 44.27 \\
\hline $\mathrm{Ce}(\mathrm{ppm})$ & 56.20 & 123.00 & 111.00 & 88.90 & 99.00 & 95.40 & 131.00 & 99.10 & 107.00 & 99.10 & 88.96 & 86.39 \\
\hline $\operatorname{Pr}(\mathrm{ppm})$ & 6.74 & 13.60 & 13.00 & 10.50 & 11.90 & 11.10 & 15.60 & 11.30 & 12.50 & 11.50 & 10.76 & 10.16 \\
\hline $\begin{array}{l}\mathrm{Nd} \\
\quad(\mathrm{ppm})\end{array}$ & 25.60 & 49.90 & 48.60 & 38.80 & 44.20 & 41.40 & 58.70 & 42.10 & 46.30 & 42.60 & 39.34 & 37.54 \\
\hline $\begin{array}{l}\mathrm{Sm} \\
\quad(\mathrm{ppm})\end{array}$ & 5.06 & 9.30 & 9.59 & 7.96 & 9.27 & 8.46 & 11.80 & 8.57 & 9.36 & 8.50 & 8.61 & 8.39 \\
\hline $\mathrm{Eu}(\mathrm{ppm})$ & 1.05 & 1.22 & 1.46 & 1.04 & 1.51 & 1.50 & 1.85 & 1.40 & 1.42 & 1.43 & 1.50 & 1.38 \\
\hline $\begin{array}{l}\text { Gd } \\
\quad(\mathrm{ppm})\end{array}$ & 4.57 & 7.76 & 8.65 & 7.23 & 9.05 & 7.64 & 10.40 & 7.76 & 8.25 & 8.65 & 8.30 & 7.55 \\
\hline $\mathrm{Tb}(\mathrm{ppm})$ & 0.68 & 1.26 & 1.32 & 1.17 & 1.50 & 1.17 & 1.54 & 1.20 & 1.22 & 1.39 & 1.34 & 1.23 \\
\hline $\begin{array}{l}\text { Dy } \\
\text { (ppm) }\end{array}$ & 4.35 & 8.84 & 8.15 & 7.50 & 9.39 & 7.67 & 9.67 & 7.53 & 7.33 & 9.14 & 8.73 & 8.21 \\
\hline $\begin{array}{l}\text { Ho } \\
\qquad(\mathrm{ppm})\end{array}$ & 0.88 & 1.95 & 1.65 & 1.53 & 1.86 & 1.58 & 2.00 & 1.51 & 1.47 & 1.87 & 1.83 & 1.63 \\
\hline $\mathrm{Er}(\mathrm{ppm})$ & 2.52 & 6.07 & 4.73 & 4.46 & 5.23 & 4.77 & 5.95 & 4.34 & 4.27 & 5.31 & 4.93 & 4.50 \\
\hline $\begin{array}{l}\mathrm{Tm} \\
\quad(\mathrm{ppm})\end{array}$ & 0.35 & 0.93 & 0.67 & 0.65 & 0.74 & 0.71 & 0.87 & 0.61 & 0.62 & 0.75 & 0.68 & 0.63 \\
\hline $\begin{array}{l}\mathrm{Yb} \\
\quad(\mathrm{ppm})\end{array}$ & 2.42 & 6.51 & 4.61 & 4.44 & 5.08 & 4.92 & 6.07 & 4.17 & 4.24 & 4.88 & 4.60 & 4.33 \\
\hline $\mathrm{Lu}(\mathrm{ppm})$ & 0.33 & 0.92 & 0.64 & 0.60 & 0.70 & 0.71 & 0.87 & 0.57 & 0.60 & 0.68 & 0.64 & 0.60 \\
\hline Hf (ppm) & 4.05 & 9.36 & 7.39 & 6.15 & 6.57 & 8.35 & 9.23 & 5.59 & 6.84 & 6.03 & 3.21 & 3.10 \\
\hline $\mathrm{Ta}(\mathrm{ppm})$ & 0.73 & 1.92 & 1.52 & 1.32 & 1.38 & 1.73 & 1.90 & 1.29 & 1.55 & 1.32 & 1.03 & 0.97 \\
\hline $\mathrm{Th}$ (ppm) & 7.53 & 19.30 & 16.00 & 14.30 & 14.30 & 16.80 & 18.90 & 12.50 & 15.90 & 13.80 & 18.50 & 17.91 \\
\hline $\mathrm{U}(\mathrm{ppm})$ & 1.60 & 3.33 & 3.44 & 2.61 & 3.13 & 3.25 & 3.97 & 2.94 & 3.26 & 3.17 & 2.94 & 2.75 \\
\hline
\end{tabular}

1 Lower Carboniferous; 2 Frasnian; 3 Eifelian; 4 Emsian (Lenne area); 5 Emsian (Taunus); 6 Silurian (Taunus-South Harz phyllite zone)

SHMS, VHMS and Lahn-Dill-type ore deposits. These three types of mineralization are characterized in the following way:

1. Sediment-hosted massive sulfide deposits (SHMS, Cu$\mathrm{Pb}-\mathrm{Zn}-\mathrm{Fe}$ sulfide deposits with various amounts of barite) of the Rhenish Massif and Harz areas (Meggen, Rammelsberg) represent a Middle Devonian evolution, which combines subsidence of local troughs with formation of black shales under anoxic conditions and input of hydrothermal fluids along supposed active faults. They would resemble conditions of Red Seatype deposits (Atlantis III) or euxinic Black Sea-type. Equivalent shale-hosted ore bodies are observed in the southern part of the Iberian Pyrite Belt (e.g., Tharsis, Neves Corvo, Aznalcollar; Tornos 2006), interpreted as anoxic smaller basins (brine pools) of Upper Devonian age with precipitation of massive sulfides at the sea floor.

2. Volcanic-hosted massive sulfide deposits (VHMS) are known from the Rhenish Massif (Lohrheim, Middle Devonian age; Kroll and Borchert 1969; Emmermann et al. 1993), from the Harz area (Einheit, Middle Devonian age), from the Moravo-Silesian units (Zlaté Horu, Horni Mesto, Horni Benesov; Middle Devonian age, Aichler et al. 1995; Large 2003a). In the northern part of the Iberian Pyrite Belt (Concepción, San Platón, Aguas Teñidas Este, San Miguel, La Zarza, Aljustrel, or Filón Norte in Rio Tinto, Tornos 2006) in contrast, they are mostly hosted by massive or volcaniclastic, vitriclast- or pumice-rich, felsic rocks of Tournaisian age. 


\section{Ma Frasnian}

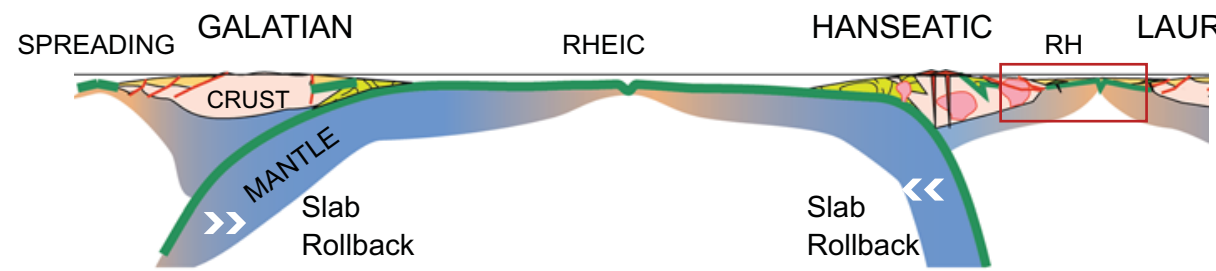

BACKARC SPREADING

PASSIVE MARGIN

RIFTING

THERMAL UPLIFT

EARLY ORE DEPOSITS

\section{Ma Famennian}

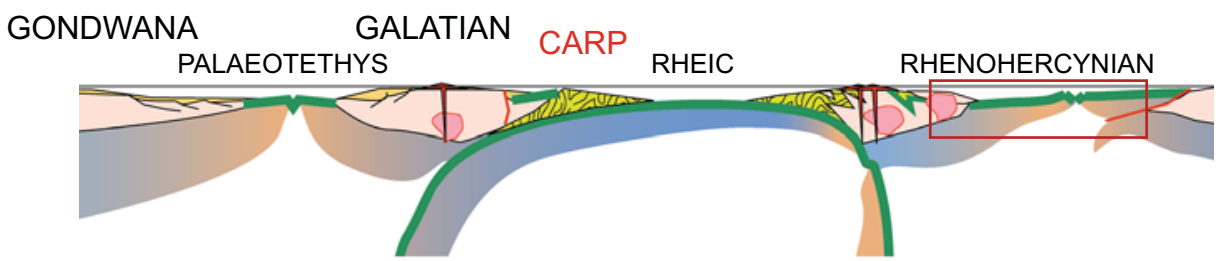

DRIFTING OF

RIBBON CONTINENTS

RIDGE SUBDUCTION

EARLY ORE DEPOSITS

\section{Ma E.Visean}

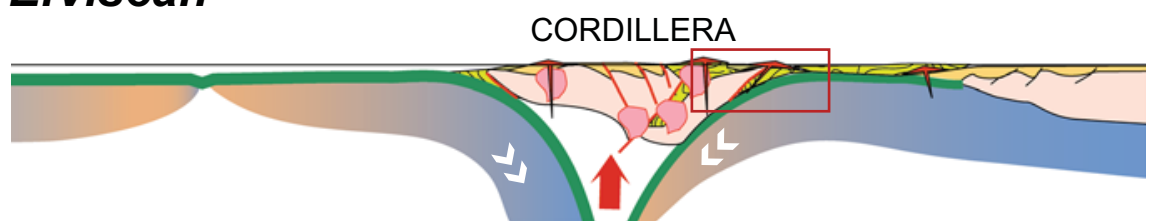

SLAB DETACHMENT

ACIDIC VOLCANICS

LATE ORE DEPOSITS

Fig. 6 Cross-section models (modified after Stampfli et al. 2011): from Frasnien back-arc spreading to Tournaisian slab detachment, emplacement of acidic volcanics and ore deposits. CARP-supposed location of Carpathian ore sites; $\mathrm{RH}-$ Rhenohercynian Ocean. Crustal and mantle levels are indicated in the 380 Ma section: man- tle domains revealing from bluish to orange tints rising temperatures. Crustal levels represent basement domains (pink) with granitoid bodies, detrital sedimentary fans (light green) and oceanic crust (dark green). Red frames indicate the location of the discussed ore deposits. Frame 370 roughly corresponds to the model of Fig. 7
3. The Lahn-Dill-type deposits in the Lahn-Dill area and in Moravia are exhalative $\mathrm{Fe}$ oxide mineralizations associated with basaltic volcanic centers. The proper Lahn-Dill-type hematitic iron ores ("Roteisenstein-Grenzlager") formed at the limit of the Middle to Upper Devonian (Lippert 1951; Lippert and Flick (1998). The formation of the iron ores by mobilization of $\mathrm{Fe}, \mathrm{Ca}$ and $\mathrm{Si}$ resulted from hydrothermal alteration and leaching of subjacent submarine basaltic volcanics (Flick et al. 1990). Calcite cements (in part with spheroidal fabric indicating replacement of aragonite) document near-neutral $\mathrm{pH}$ conditions. Ore deposition is interpreted as the result of precipitation of iron oxides and hydroxides when near-neutral and reduced Fe-bearing hot fluids encounter cool oxidizing water. The iron deposit of the "Schalstein-Lager" were deposited in pools on the floor of the shelf sea. Local reducing conditions and availability of reduced sulfur explain the formation of the sporadic sulfide rich deposits.

Supposed to have been formed at or just beneath the sea floor during the pre-orogenic (Middle to Upper Devonian) basin development (Large 2003b), the exhalative SHMS mineralizations are associated with tectonic and igneous activity during the post-rift or "sag"-phase of basin evolution. Preconizing comparable geochemistry and isotope characteristics, Aichler et al. (1995) and Werner and Walther (1995) supposed the precipitation from hydrothermal brines for the Rhenish and Bohemian ore sites having their origin in Middle Devonian syn-rift shelf sediments. The presence of sulfidic framboids testifies reducing conditions (presence of organic matter; Love and Amstutz 1966; Sperling 1986), conditions typical for organic matter in sediments. In the southern part of the Iberian Pyrite Belt (Tornos 2006), upwelling deep sulfur-depleted fluids mixed with modified seawater rich in biogenically reduced sulfur lead to the precipitation of massive sulfides, sulfidic framboids being frequent as well (Menor et al. 2010). Such deposits of mostly stratiform morphology with abundant sedimentary structures were formed in the uppermost Devonian. They are supposed to represent a short epoch of vigorous hydrothermal activity, favoring the development of stable hydrothermal systems (Figs. 6, 7).

In contrast, the ore sites of the northern part of the Pyrite belt formed as volcanic-hosted Tournaisian felsic-clastic deposits in a pull-apart basin, interpreted by Tornos (2006) as stratabound ores which precipitated by mixing of deep 


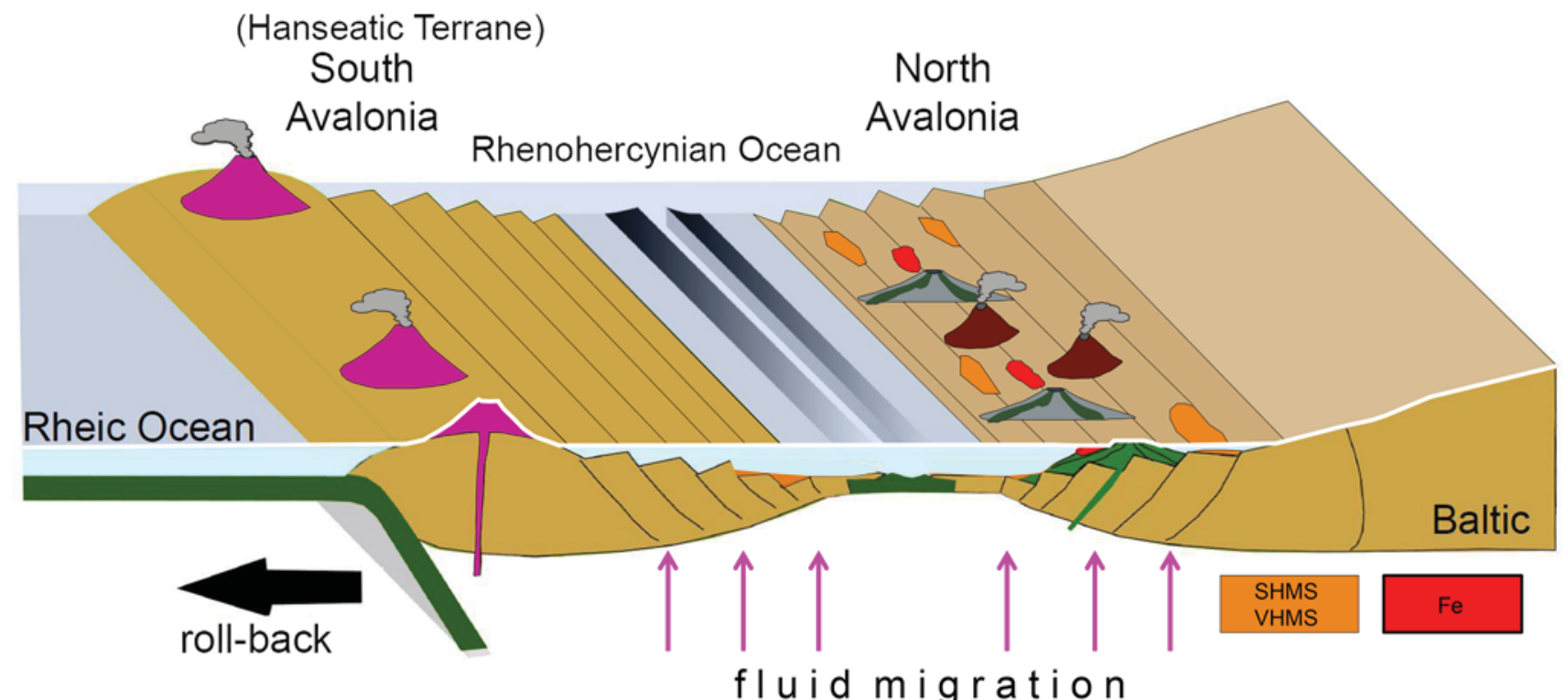

Fig. 7 Model for the possible tectonic setting during Middle to Late Devonian SHMS (Riotinto S, Meggen, Rammelsberg), VHMS (Einheit in the Harz area, Zlaté Hory, Horni Mesto, Horni Benesov) and Lahn-Dill ore deposits. They are interpreted to have formed in an initial back-arc-type extensional setting above the subducting Rheic Ocean. Purple cones: subduction related felsic volcanoes; grey cones:

fluids with modified seawater and variably reduced sulfur, compatible with the dominant derivation of the deep fluids and metals from the underlying siliciclastic PQ Group of Upper Devonian age. Menor-Salván et al. (2010) invoke a temporal and causal relationship between the DevonianCarboniferous geotectonic, climatic and biological crises and the formation of the giant volcano-sedimentary massive sulfide deposits of the southern Iberian Pyrite Belt.

\section{The Devonian plate-tectonic environment-a discussion}

The plate-tectonic evolution of the Variscan areas has been hitherto represented in various geological maps and corresponding cross sections. However, the related difficulties become specifically evident, when considering the appreciation of the South Portuguese sections, hosting the well-known Rio Tinto ores. The evolution through time and complex juxtaposition of advancing Variscan slivers of pre-Variscan basement and its cover (Quesada 1998, 2006) could not be resolved simply by reconstructing a pre-existing paleogeographical situation for one given time period. Equally, it is not apparent from Franke's (2000) interpretation of the detrital input during the Variscan tectonic evolution, whether a possible lack of surface information would not hinder a different interpretation. The non-uniform

intraplate basaltic volcanoes with lava flows (green); dark red cones: intraplate felsic volcanoes. Geology; geology modified after Eckelmann et al. (2014b). The model roughly corresponds to the location indicated in cross section $370 \mathrm{Ma}$ in Fig. 6. Orange: location of Devonian ore sites, all ore sites being projected at surface; red: indication of iron ores projected at surface

subsidence in the "rapidly subsiding ... asymmetrical graben-like structures" of the Rhenohercynian domain (Walliser 1981, p. 89), and their related geometric complications could inverse the reconstruction of the detrital input.

An amplified view resulted from considering the geodynamic evolution in distinct time sections. Not easy to reconstruct, when considering the plate-tectonic complications during Devonian to Carboniferous times (Franke 2000; Oncken et al. 2000), Stampfli et al. (2002) presented already the opening of the Rhenohercynian Ocean as a stepwise evolution from a simple rift on the Avalonian shelf, evolving subsequently to the opening of the Rhenohercynian Ocean, the local separation of slivers of the Avalonian basement domain (Hanseatic terrane, Stampfli et al. 2011, their Fig. 7) included. The subsequent collisional evolution for the Rhenohercynian Zone has been well characterized by Oncken et al. (2000).

Floyd (1982), Floyd et al. (1983) and Ziegler (1988) interpreted the evolution of the Rhenohercynian structure as a back-arc rift zone, whereas Oncken et al. (1999), instead, saw the evolution of a failed Devonian rift, where "peak crustal stretching occurred during the Late Lower Devonian, recorded by strong subsidence, formation of growth faults and widespread volcanism" (Oncken and Weber 1995, p. 50). Reliable arguments for early continental rifting were produced by Stets and Schäfer (2009, 2011), Nesbor (2004), Salamon (2008) and Salamon and 
Königshof (2010). Using the distribution of detrital zircons, Eckelmann et al. (2014b) reconstructed the tectonic puzzle by a scenario, including a north-subducting northern limit of the Rheic Ocean during the Devonian. The possible relation of the north-directed slab of the Rheic Ocean subducting under the initial rift structure of the Rhenohercynian trough would represent a reliable explanation for correlating the subducting oceanic crust with the subsequent formation of diverse ore types in the adjacent Rhenohercynian Zone.

If presenting the evolution of the Rhenohercynian ore sites in this general plate-tectonic aspect (comp. Figure 3), the timing of Early Devonian crustal extension within the Mid-German Crystalline Rise was already preceded during the Silurian by an active margin setting by the development of a magmatic arc, as documented in the Taunus-South Harz phyllite zone (Sommermann et al. 1992, 1994) and in the Spessart and Böllsteiner Odenwald areas (Dombrowski et al. 1995; Anthes and Reischmann 2001; Reischmann and Anthes 1996; Reischmann et al. 2001). The continuity of this arc in Devonian times is proven by radiometric datings in the Böllsteiner Odenwald, with the result of Late Upper Devonian protolith ages of orthogneisses (Reischmann et al. 2001).

In reconstructing the Variscan tectonic puzzle, valuable information by Huckriede et al. (2004) attributes the input of detrital white micas for the Rhenohercynian autochthonous sediments east of the river Rhine to orogenic events, where most of the flysch sediments reveal Early Variscan cooling ages. Demonstrating the erosion of metamorphic basement before their Late Devonian to Carboniferous deposition, non-flysch sediments were shown to have principally two provenances: either Caledonian (440-410 Ma) being widespread in the larger domain or a Cadomian (650-550 Ma) age group, showing affinities to the Saxothuringian domain (Huckriede et al. 2004). The latter could represent, instead, the relics of a former rift shoulder of the southern border of the Laurasian continent before the opening of the Rhenohercynian Ocean (named Hanseatic terrane by Stampfli et al. 2011).

More recently, precision from zircon age data (Eckelmann et al. 2014a, b) differentiated in provenances and fossil assemblages between autochthonous to par-autochthonous and allochthonous units. Suggesting differences of spatial separation of detrital sources during the Devonian and the Lower Carboniferous, such observations suppose distinct oceanic domains for specific time intervals. Eckelmann et al. (2014a, b) concluded that the Rheic Ocean lasted from Silurian to Visean times, subducting northward under Laurussia and triggering the opening of the Rhenohercynian Ocean in a back-arc position. This proposal confirms the existence of a north-subducting branch of the Rheic Ocean (Von Raumer and Stampfli 2008; Zeh and
Gerdes 2010) and influences considerably on the trace of the Variscan collision in Central Europe (Von Raumer et al. 2013).

The closure of all oceanic domains is visible in the flysch sedimentation (Culm Greywacke), whose source areas show an Armorican provenance, generally.

Similarly, in the Central Iberian-South Portuguese area, zircon age data from detrital zircons (Pereira et al. 2012) reveal active wrench tectonics including exhumation and basin instability during the Early Carboniferous, flysch sediments being recognized over large domains. Although the Pyrite belt is not specifically discussed in this strongly juxtaposed assemblage (Pereira et al. 2012), the attribution of part of this assemblage to the Rhenohercynian type of evolution at the Laurussian border should not be disregarded (see discussion above). Menor-Salván et al. (2010) indicate well the complication of the general plate-tectonic situation, where the closure of the Rheic Ocean between the Hanseatic terrane and Ossa Morena-type microcontinents (Stampfli et al. 2013), the supposed detachment of the Rheic slab (von Raumer et al. 2015) and the subsequent subduction reversal (Stampfli et al. 2013), would accelerate an orographic evolution, and a strong mobilization of thermal circulation.

The new observations (Eckelmann et al. 2014a, b) for the Rhenish part of the Rhenohercynian Zone need discussion and lead to reformulate established models, before correlating the different events influencing ore deposition. A wide Rheic Ocean through the Devonian with a northward dipping subduction zone below Laurussia associated with a back-arc setting was discussed largely (Floyd 1982; Flick and Nesbor 1988; Oczlon 1994; Smith 1996; von Raumer and Stampfli 2008; Zeh and Gerdes 2010, Stampfli et al. 2013). The argumentation for such a large ocean during the regarded time range is the strict separation between Baltica/Avalonia and Armorica source areas of sediments in the autochthonous and allochthonous units, respectively. No overlap or mixing of the erosion product from these domains was identified, yet. The existence of a subduction zone at the southern margin of Laurussia is documented by typical subduction-related volcanic products in the Rhenohercynian and the corresponding plutonic rocks in the northern part of the Mid-German Crystalline Zone. But local differences may appear, when considering the two-plate Variscan plate-tectonic evolution of the Saxothuringian Zone (Kroner et al. 2007), juxtaposing the Rhenohercynian Zone and its respective ore sites toward the south (see discussion, Will et al. 2015).

The volcanic activity is documented in numerous ash layers of dacitic/rhyodacitic and rhyolitic composition intercalated within the sedimentary rock pile of the Rhenohercynian (Heyckendorf 1985; Kubanek and Zimmerle 1986; Kirnbauer 1991; van Amerom et al. 2001). This kind 
of silica-rich volcanism is characteristic for a thick continental crust above the subducting slab. Beside the uniform composition of the magmas, the long-lasting volcanic activity of about 50-60 Ma is remarkable, requiring a constant process of melt generation, which only can be realized in a subduction setting of a large ocean.

To support our interpretation in this context, a closer view on the Silurian volcanism in the Taunus-South Harz phyllite zone ("Northern Phyllite Zone") provide further information. The melt generation of these volcanics in a subduction-related setting is generally accepted (e.g., Franke and Oncken 1995; Franke 2000). Results of petrological and geochemical investigations have shown that the element pattern of the Silurian rhyolites and those of the Devonian is identical (Figs. 4, 5). For both volcanic units, equal conditions of an overall presence of a transformed high-grade Cadomian basement with a former history as part of a magmatic arc (peri-Gondwana) was described, not needing to interpret the same rock type in one case as subduction-related and in the other case as recycled material of older arc material.

Interestingly, new data from the Mid-German Crystalline Rise (Will et al. 2015) bring convincing arguments from chemical data on amphibolites that back-arc-type metabasic rocks appear on the Gondwana side in the western Odenwald and southern Spessart, whereas the northern Spessart domain exposes intraplate-type metabasites, supposedly belonging to the Avalonian side of basement, appearing window-like below the overthrusted back-arc magmatites. This situation would correspond to the given eclogite ages, pleading also for a location on the suture Baltica-Avalonia, when Avalonia began to collide with Baltica (comp. Stampfli et al. 2013, their Fig. 3), corresponding to a comparable section in the French vicinity (Will et al. 2015, their Fig. 1), which would represent a location north of the Leon domain.

Amstutz et al. (1971) correlated the Middle Devonian massive sulfides and Lahn-Dill-type mineralizations from the southern and eastern Carpathians (Kräutner 1970) with those of the Rhenohercynian domain. Although Gawęda et al. (2016) plead for a subduction environment under a globally called "Rhenohercynian Zone" for the western Carpathian Tatra region and its granitoids, their location in the eastern continuation of the Eastern Alps (Adriatic plate, Schulz et al. 2008) would not coincide with Kräutner's (1970) supposed ore distribution (comp. Handy et al. 2010). The southern Carpathian Padés and Fagaras terranes (Balintoni et al. 2014), hosting the Pojana Rusca ore site, are characterized by Middle Devonian sideritic iron ores deposited in a volcano-sedimentary diabase-spilite formation (Kräutner 1970), whereas Kräutner's (1970) ore sites from the eastern Carpathians would correspond to the Rebra and Tulghes terranes in Balintoni et al. (2014).
The rifting of the Rhenohercynian and Palaeotethys oceans occurring nearly at the same time, the Devonian timing of Pojana Ruska (394 Ma; Kohut et al. 2006) would likewise coincide with the tectonic evolution of the Palaeotethys domain in Balintoni et al. (2014). Consequently, Kräutner's (1970) Carpathian localities could be correlated with the subduction of the south-directed slab of the Rheic Ocean (as explained in Figs. 3, 6 and Stampfli et al. 2013), before the Carpathian basement structures disappeared in the Variscan collision during Visean times.

How could the local occurrences of Rhenohercynian ore sites be interpreted? Large (2003b, his Fig. 7) proposed pre-orogenic extension (Lower Devonian "syn-rift") triggering syn-sedimentary basic volcanics and leading to the Middle to Upper Devonian "post-rift" ore sites. In a scenario of Lower to Middle Devonian oblique collision (Stampfli et al. 2002, Fig. 4; Stampfli et al. 2013) the presence of pull-apart structures could easily explain the local occurrence of massive sulfide ores (SHMS). However, overprint or leaching of hitherto unknown preexisting metal concentrations and their effect requires future more specialized research.

\section{Summary-conclusion}

Considering the Devonian plate-tectonic environment, differences appear when appreciating the plate-tectonic evolution. In the general juxtaposition of terrane assemblages during Devonian times, formerly being part of a long ribbon continent behind the subducting Rheic Ocean, Matte (2001) admitted an iteration of oceanic spaces and microplates, like the opening Rheic Ocean and the Mid-European Galician Ocean. Stampfli et al. (2013, their Fig. 7), instead, interpreted the presence of multiple sutures as the Devonian duplication of the Rheic suture through strike-slip and rotation of blocks, thus allowing to find the many pieces of the former subduction zone (relics of eclogite) of the Rheic Ocean across the Variscan basements of Europe (e.g., Lardeaux et al. 2001; Stampfli et al. 2002), and characterising the Variscan collision zone across Europe before the general Visean thermal evolution (von Raumer et al. 2013).

In this general scenario, the coincidence of several platetectonic processes produced an environment appropriate for ore precipitation. The large shelf of the Devonian Old Red continent was the place of thermal subsidence with contemporaneous mobilization of rising thermal fluids along activated Early Devonian growth faults. Subductionrelated volcanism and intraplate volcanic activity are seen as contemporaneous processes during crustal extension in a back-arc regime.

We consequently conclude that mostly during Middle to Late Devonian hydrothermal brines equilibrated with the 
basement and the overlying Givetian-Fammenian detrital deposits and volcanics. Exhaling on the seafloor, they produced brine pools and mineralizations through $\mathrm{H}_{2} \mathrm{~S}$ input from biogenic reduction.

In contrast, since the Tournaisian period of ore formation, dominant pull-apart triggered magmatic emplacement of acidic rocks and lead to metasomatic replacement in the apical zones of felsic domes and sediments, thus changing the general conditions of ore precipitation.

The question remains what are the causes of the rather spotwise appearance of the base metal mineralizations in the Rhenohercynian Zone. Were they only related to local thrusts (Large 2003a, b; Aichler et al. 1995) or were they triggered by locally appearing pull-apart structures since the Early Devonian? Do they testify for the thermal consequences of former Late Ordovician to Silurian volcanic arcs? Or could they even represent the detrital relics of older, yet unknown sources, which were dispersed in the Devonian detritus? Future isotope geochemical research would certainly help to find the answers.

Acknowledgements We appreciated the thoughtful suggestions and critical remarks from Lluis Fonboté (Geneva) and an unnamed reviewer, and we enjoyed the discussions with J Balintoni (Cluj), F Finger (Salzburg), H Flick (Heidelberg), D Large (Braunschweig), F Neubauer (Salzburg), A Schaefer (Bonn), B Schulz (Freiberg) and F Tornos (Madrid). G. Wörner (Göttingen) is thanked for performance of the geochemical analyses. We appreciated the understanding help from W C Dullo (Editor-in-Chief).

\section{References}

Aichler J, Fojt B, Vanecek M (1995) Metallogenesis. In: Dallmeyer RD, Franke W, Weber K (eds) Pre-Permian geology of central and eastern Europe. Springer, Berlin, pp 512-517

Amstutz GC, Zimmermann RA, Schot EH (1971) The Devonian mineral belt of Western Germany (The mines of Meggen, Ramsbeck and Rammelsberg), VIII. International Sedimentology Congress. 253-272

Anthes G, Reischmann T (2001) Timing of granitoid magmatism in the eastern mid-German crystalline rise. J Geodyn 31:119-143

Balintoni I, Balica C, Ducea MN, Hann H-P (2014) Peri-Gondwanan terranes in the Romanian Carpathians: a review of their spatial distribution, origin, provenance, and evolution. Geosci Front 5:395-411

Becker Th (2008) Devonian neritic-pelagic correlations-methods, case studies and problems. Final Meeting of IGCP 497 and 499. Abstract. In: Königshof P, Linnemann U (eds) From Gondwana and Laurussia to Pangaea: dynamics of oceans and supercontinents, 20th International Senckenberg Conference, Frankfurt, September 30-October 3, Abstracts and Programme, pp 25-28

Dehmer J, Hentschel G, Horn M, Kubanek F, Nöltner T, Rieken R, Wolf M, Zimmerle W (1989) Die vulkanisch-kieselige Gesteinsassoziation am Beispiel der unterkarbonischen Kieselschiefer am Ostrand des Rheinischen Schiefergebirges. Geologie-Petrographie-Geochemie. Geol JB Hessen 117:79-138

Dombrowski A, Henjes-Kunst F, Höhndorf A, Kröner A, Okrusch M, Richter P (1995) Orthogneisses in the Spessart crystalline complex, northwest Bavaria: Silurian granitoid magmatism at an active continental margin. Geol Rundsch 84:399-411
Doublier MP, Potel S, Franke W, Roache T (2012) Very low-grade metamorphism of Rheno-Hercynian allochthons (Variscides, Germany): facts and tectonic consequences. Int J Earth Sci 101:1229-1252

Eckelmann K, Linnemann U, Bahlburg H, Jansen U, Königshof P, Nesbor H D, Gerdes A, Hofmann M, Berndt J, Nawrat J (2014a) The provenance of exotic Ordovician and Devonian sedimentary rock units in the Lindener Mark (SE Rhenich Massif), Central EuropeanVariscides, Germany)—a combined $\mathrm{U}-\mathrm{Pb}$ and $\mathrm{Hf}$ isotope study of detrital zircons. Abstract. Gondwana 15-North meets South, Madrid (Spain) 14-18 July, 2014. Abstract Vol p. 56

Eckelmann K, Nesbo H D, Königshof P, Linnemann U, Hofmann M, Lange JM, Sagawe A (2014b) Plate interactions of Laurussia and Gondwana during the formation of Pangaea-Constraints from U-Pb LA-SF-ICP-MS detrital zircon ages of Devonian and Early Carboniferous siliciclastics of the Rhenohercynian zone, Central European Variscides. Gondwana Res 1484-1500

Emmermann KH, Hindel R, Krimmel M, Zinner HJ, (1993) SulfidBaryt-Mineralisationen in der südwestlichen Lahnmulde. Ein Beitrag zur Genese und Prospektion synsedimentär-exhalativer Vorkommen im Rheinischen Schiefergebirge. Mainzer geowissenschaftliche Mitteilungen, 22: 7-38

Flick H, Nesbor HD (1988) Der Vulkanismus in der Lahnmulde. Jb Mitt Oberrh Ver N F 70:411-475

Flick H, Nesbor HD, Behnisch R (1990) Iron ore of the Lahn-Dill type formed by diagenetic seeping of pyroclastic sequencesa case study on the Schalstein section at Gänsberg (Weilburg). Geol Rdsch 79:401-415

Floyd PA (1982) Chemical variation in Hercynian basalts relative to plate tectonics. J Geol Soc Lond 139:505-520

Floyd PA, Exley CS, Stone M (1983) Variscan magmatism in southwest England-discussion and synthesis. In: Hancock PL (ed) The variscan fold belt in the British Isles. Taylor \& Francis, Bristol, pp 178-185

Franke W (1989) Tectonostratigraphic units in the variscan belt of central Europe. Geol Soc Am Spec PAPER 290:67-89

Franke W (1995) Rhenohercynian foldbelt: autochthon and nonmetamorphic nappe units-stratigraphy. In: Dallmeyer D, Franke W, Weber K (eds) Pre-Permian geology of Central-and Western Europe. Springer, Berlin, pp 33-49

Franke W (2000) The mid-European segment of the Variscides: Tectonostratigraphic units, terrane boundaries and plate tectonic evolution. In: Franke W, Haak V, Oncken O, Tanner D (eds) Orogenic processes-quantification and modelling in the variscan belt of central Europe. Geol Soc London Sp Pub 170, 459 p

Franke W, Engel W (1986) Synorogenic sedimentation in the Variscan belt of Europe. Bulletin de la Societé géologique de France, 8(II1):25-33

Franke W, Oncken O (1995) Zur prä-devonischen Geschichte des Rhenoherzynischen Beckens. Nova Acta Leopoldina NF 71:53-72

Frischmuth E (1968) Sedimentation und Tektonik in der Subvariszischen Vortiefe von Süd-Portugal. Münstersche Forschungen zur Geologie und Paläontologie. Vol. 4, 99 p, Münster

Gawęda A, Burda J, Klötzli U, Golonka J, Szopa K (2016) Episodic construction of the Tatra granitoid intrusion (Central Western Carpathians, Poland/Slovakia): consequences for the geodynamics of Variscan collision and Rheic Ocean closure. Int J Earth Sci (Geol Rundsch). doi:10.1007/s00531-015-1239-2

Handy MR, Schmid DM, Bousquet R, Kissling E, Bernoulli D (2010) Reconciling plate-tectonic reconstructions of Alpine Tethys with the geological-geophysical record of spreading and subduction in the Alps. Earth Sci Rev 102:121-158. doi:10.1016/j. earscirev.2010.06.002

Herbig HG, Aretz M (2013) The Mississippian of the Jerada massif (NE Morocco)-more northwest European than Palaeotethyan 
affinities. Schriftenreihe der Deutschen Gesellschaft für Geowissenschaften SDGG 82:50

Heyckendorf K (1985) Die unterdevonischen Lenne-Vulkanite im nordöstlichen Rheinischen Schiefergebirge: Beiträge zur Stratigraphie, Paläogeographie, Petrographieund Geochemie. Mitteilungen aus dem Geologisch-Paläontologischen Institut der Universität Hamburg 68: 1-52

Hochard C (2008) GIS and geodatabases application to global scale platetectonics modelling. PhD Thesis, University of Lausanne, Switzerland

Huckriede H, Wemmer W, Ahrendt H (2004) Palaeogeography and tectonic structure of allochthonous units in the German part of the Rheno-Hercynian Belt (Central European Variscides). Int J Earth Sci (Geol Runsch) 93:414-431

Hüneke H (2007): Pelagic carbonate ooze reworked by bottom currents during Devonian approach of the continents Gondwana and Laurussia. In: Viana AR, Rebesco M (eds.) Economic and palaeoceanographic significance of contourite deposits. Geol Soc London Spec Publ 276:299-328

Hüneke H (2008) Formation of Givetian-Frasnian contourites in pelagic and hemipelagic settings prior to the virtual suturing of Gondwana and Laurussia: clues to palaeocirculation and continental reconstruction. Final Meeting Of IGCP 497 and 499. Abstract. In: Königshof P, Linnemann U (eds) From Gondwana and Laurussia to Pangaea: Dynamics of oceans and supercontinents, 20th international senckenberg conference, Frankfurt, September 30-October 3, Abstracts and Programm, pp. 54-55

Kirnbauer T (1991) Geologie, Petrographie und Geochemie der Pyroklastika des Unteren Ems/Unterdevon (Porphyroide) im südlichen Rheinischen Schiefergebirge. Geol Abh Hess 92:1-228

Kohut M, Konecny P, Siman P (2006) The first finding of the iron Lahn-Dill mineralization in the Tatric Unit of the Western Carpathians. Mineral Polon-Spec Pap 28:112-114

Königshof P, Nesbor HD, Flick H (2010) Volcanism and reef development in the Devonian: a case study from the Lahn syncline, Rheinisches Schiefergebirge (Germany). Gondwana Res 17:264-280

Kräutner HG (1970) Die hercynische Geosynklinalerzbildung in den rumänischen Karpaten und ihre Beziehungen zu der hercynischen Metallogenese Mitteleuropas. Miner Deposita 4:323-344

Kroll JM, Borchert W (1969) Geologisch-petrographische Untersuchungen an westdeutschen Kaolinlagerstätten-II. Kaolinlagerstätte Lohrheim bei Diez an der Lahn. Berichte Deutsche Keramische Gesellschaft 46:81-85

Kroner U, Hahn T, Romer R L, Linnemann U (2007) The variscan orogeny in the saxo-thuringian zone-heterogenous overprint of cadomian/paleozoic peri-gondwana crust. In: Linnemann U, Nance RD, Kraft P, Zulauf G (eds) The evolution of the Rheic Ocean: from Avalonian-Cadomian active margin to AlleghenianVariscan collision. Geol Soc Am Spec Pap 423, 153-172

Kubanek F, Zimmerle W (1986) Tuffe und kieselige Tonschiefer aus dem tieferen Unterkarbon der Bohrung Adlersberg (West-Harz). Geol Jahrb D 78:207-268

Lardeaux JM, Ledru P, Daniel I, Duchene S (2001) The Variscan French Massif Central-a new addition to the ultrahigh pressure metamorphic "club": exhumation processes and geodynamic consequences. Tectonophys 332:143-167

Large DE (2003a) Base metal (Cu, Pb, Zn) metallogeny of Europe-an overview. In: Kelly JG, Andrew CJ, Ashton MB, Boland MB, Earls G, Fusiardi L, Stanley G (eds) Europe's major base metal deposits. Irish Association for Economic Geology, Dublin, pp 1-29

Large DE (2003b) The tectonic setting of base-metal mineralisation in the Rhenohercynian terranes of central Europe. In: Kelly JG, Andrew CJ, Ashton MB, Boland MB, Earls G, Fusiardi L, Stanley $\mathrm{G}$ (eds) Europe's major base metal deposits. Irish Association for Economic Geology, Dublin, pp 155-168
Lippert H-J (1951) Zur Gesteins-und Lagerstättenbildung in Roteisenstein-Gruben des östlichen Dill-Gebietes. Abh Senckenb Naturforsch Ges, 485 (R. Richter-Festschrift). 1-29

Lippert H-J, Flick H (1998) Vulkano-sedimentäre Roteisenerze vom Lahn-Dill-Typ. In: Kirnbauer T (ed) Geologie und hydrothermale Mineralisationen im rechtsrheinischen Schiefergebirge. Nassauischer Verein für Naturkunde, Wiesbaden, Sonderband 1, $121-128$

Love LG, Amstutz GC (1966) Review of microscopic pyrite from the Devonian Chattanooga Shale and Rammelsberg Banderz. Fortschr Mineral 43:273-309

MacDonald WF, Sun S (1995) The composition of the Earth. Chem Geol 120:223-253

Martínez Catalán JR (2012) The Central Iberian arc, an orocline centered in the Iberian Massif and some implications for the Variscan belt. Int J Earth Sci (Geol Rundsch) 101:1299-1314

Matte P (2001) The variscan collage and orogeny (480-290 Ma) and the tectonic definition of the Armorica microplate; a review. Terra Nova 13:122-128

Meisl S (1995) Igneous activity. In: Dallmeyer RD, Franke W, Weber $\mathrm{K}$ (eds) Pre-permian geology of central and eastern Europe. Springer, Berlin, pp 118-131

Menor-Salván C, Tornos F, Fernández-Remolar D, Amils R (2010) Association between catastrophic paleovegetation changes during Devonian-Carboniferous boundary and the formation of giant massive sulfide deposits. Earth Planet Sci Lett 299:398-408

Nesbor HD (1997) Petrographie der vulkanischen Gesteine. In: Bender P, Lippert HJ, Nesbor HD (eds) Geologische Karte von Hessen 1:25,000, Blatt 5216 Oberscheld, 2nd edn. Erläuterungen. Hessisches Landesamt für Bodenforschung, Wiesbaden, pp 159-207

Nesbor HD (2004) Paläozoischer Intraplattenvulkanismus im östlichen Rheinischen Schiefergebirge-Magmenentwicklung und zeitlicher Ablauf. Geologisches Jahrbuch Hessen 131:145-182

Nesbor HD (2007) Paläozoischer Vulkanismus im Lahn-DillGebiet—südliches Rheinisches Schiefergebirge (Exkursion E am 12 April 2007). Jahresberichte und Mitteilungen des Oberrheinischen Geologischen Vereins, Neue Folge 89:193-216

Nesbor HD, Buggisch W, Flick H, Horn M, Lippert HJ (1993) Vulkanismus im Devon des Rhenoherzynikums. Fazielle und paläogeographische Entwicklung vulkanisch geprägter mariner Becken am Beispiel des Lahn-Dill-Gebietes. Geol Jahrb Hessen 98:3-87

Oczlon MS (1992) Examples of Palaeozoic Contourites, Tempestites and Turbidites-Classification an Palaeogeographic Approach. Heidelberger Geowissenschaftliche Abhandlungen, 53: 57-159

Oczlon MS (1994) North Gondwana origin for exotic Variscan rocks in the Rhenohercynian zone of Germany. Geol Rundsch $83: 20-31$

Oliveira JT (1990) South Portuguese zone: introduction. Stratigraphy and synsedimentary tectonism. In: Dallmeyer RD, Martínez GE (eds) PreMesozoic geology of iberia. Springer Verlag, Berlin, pp 333-347

Oliveira JT, Horn M, Paproth E (1979) Preliminary note on the stratigraphy of the Baixo Alentejo Flysch Group, Carboniferous of Southern Portugal and on the palaeographic development, compared to corresponding units in Northwest Germany. Commun Serv Geol Portugal 65:151-168

Oncken O, Weber J (1995) The structure of the Rhenohercynian. In: Dallmeyer RD, Franke W, Weber K (eds) Tectonostratigraphic evolution of the Central and East European orogens. Springer, Berlin, Heidelberg, pp 50-58

Oncken O, von Winterfeld C, Dittmar U (1999) Accretion and inversion of a rifted passive margin - the Late Paleozoic Rhenohercynian fold and thrust belt. Tectonics 18:75-91

Oncken O, Plesch A, Weber J, Ricken W, Schrader S (2000) Passive margin detachment during arc-continent collision (Central European 
Variscides). In: Franke W, Haak V, Oncken O, Tanner D (eds). Orogenic processes-quantification and modelling in the variscan belt of central Europe. Geol Soc London Sp Pub 129: 199-216

Pereira MF, Chichorro M, Brandão Silva J, Ordóñez-Casado B, Lee JKW, Williams Ian S (2012) Early carboniferous wrenching, exhumation of high-grade metamorphic rocks and basin instability in SW Iberia: constraints derived from structural geology and U-Pb and 40Ar-39Ar geochronology. Tectonophys 558-559:28-44

Quesada C (1998) A reappraisal of the structure of the Spanish segment of the Iberian Pyrite Belt. Miner Deposita 33:31-44

Quesada C (2006) The Ossa-Morena Zone of the Iberian Massif: a tectonostatigraphic approach to its evolution. $\mathrm{Z}$ dt Ges Geowiss 157:585-595

Reischmann T, Anthes G (1996) Geochronology of the mid-German crystalline rise west of the River Rhine. Geol Rundsch 85:761-774

Reischmann T, Anthes G, Jaeckel P, Altenberger U (2001) Age and origin of the Böllsteiner Odenwald. Mineral Petrol 72:29-44

Ribeiro A (1996) SW-Iberia. In: Gee DG, Zeyen HJ (eds) Lithosphere dynamics: origin and evolution of continents. Europrobe 1996 ESF Research programme. Uppsala Univrsity, pp: 90-94

Salamon M (2008) Middle Devonian olisthostromes (debrites) in the eastern Rheinish massiv-evidence for an active rifting of the Rhenic Ocean. Abstract. Final Meting of IGCP 497 and IGCP 499, Frankfurt, September 30-October 3, Abstract Volume, pp. 124-127

Salamon M, Königshof P (2010) Middle Devonian olistostromes in the Rheno-Hercynian (Rheinisches Schiefergebirge)—an indication of back arc rifting on a passive margin of Laurussia? Gondwana Res 17:281-291

Scherp A (1983) Unterdevonische Schmelztuffe im rechtsrheinischen Schiefergebirge. Neues Jb Geol Paläontol Monat 1983:47-58

Schulz B, Steenken A, Siegesmund S (2008) Geodynamic evolution of an Alpine terrane- the austroalpine basement to the south of the Tauern Window as a part of the Adriatic plate (eastern Alps). In: Siegesmund S, Fügenschuh B, Froitzheim N (eds.) Tectonic aspects of the alpine-dinaride-carpathian system. Geol Soc Lond Spec Pub 298, 5-44

Silva JB, Oliveira JT, Ribeiro A (1990) Structural outline. In: Dallmeyer RD, Martínez GE (eds) PreMesozoic geology of iberia. Springer Verlag, Berlin, pp 348-362

Smith AG (1996) Some aspects of the Phanerozoic paleogeographic evolution of Europe. Zeitschrift der Deutschen Geologischen Gesellschaft 147:147-168

Sommermann AE, Meisl S, Todt W (1992) Zirkonalter von 3 verschiedenen Metavulkaniten aus dem Südtaunus. Geol Jahrb Hess 120:67-76

Sommermann AE, Anderle HJ, Todt W (1994) Das Alter des Quarzkeratophyrs der Krausaue bei Rüdesheim am Rhein (Bl 6013 Bingen, Rheinisches Schiefergebirge). Geol Jahrb Hess 122:143-157

Sperling H (1986) Das Neue Lager der Blei-Zink-Erzlagerstätte Rammelsberg. Geol Jahrb D 85:5-177

Stampfli GM, von Raumer JF, Borel G (2002) The Paleozoic evolution of pre-Variscan terranes: FROM Gondwana to the variscan collision. In: Martínez Catalán JR, Hatcher RD Jr, Arenas R,
Díaz Garcia F (eds) Variscan-appalachian dynamics: the building of the late paleozoic basement. Geol Soc Am Spec Pap 364: 263-280

Stampfli GM, Hochard C, von Raumer JF (2006) Reconstructing the Palaeozoic Gondwana margin and its redistribution: new aspects: Geophys Res Abstr, 8, EGU06-A-02708

Stampfli GM, von Raumer JF, Wilhem C (2011) The distribution of Gondwana derived terranes in the early Paleozoic. In: Gutiérrez Marco JC, Rábano I, García-Bellido I (eds) The ordovician of the world. Instituto Geológico y Minero de España, Madrid, Cuadernos del Museo Geominero, 14: 567-574

Stampfli GM, Hochard C, Vérard C, Wilhem C, von Raumer JF (2013) The geodynamics of Pangea formation. Tectonophys 593:1-19

Stets J, Schäfer A (2009) The Siegenian delta: land-sea transitions at the northern margin of the Rhenohercynian Basin. In: Königshof $\mathrm{P}$ (ed) Devonian change: case studies in palaeogeography and palaeoecology. Geol Soc Lond Spec Publ 314: 37-72

Stets J, Schäfer A (2011) The lower devonian rhenohercynian rift $20 \mathrm{Ma}$ of sedimentation and tectonics (Rhenish Massif, NWGermany). Z dt Ges Geowiss, 162: 93-115

Tornos F (2006) Environment of formation and styles of volcanogenic massive sulfides: the Iberian Pyrite Belt. Ore Geol Rev 28:259-307

Van Amerom HWJ, Heggemann H, Herbig HG, Horn M, Korn D, Nesbor HD, Schrader S (2001) Das Grauwacken-Profil (OberViseum) des Steinbruchs Dainrode im Kellerwald (NW Hessen). Geol Jahrb Hess 129:5-25

Von Raumer J, Stampfli GM (2008) The birth of the Rheic Oceanearly Palaeozoic subsidence patterns and tectonic plate scenarios. Tectonophys 461:9-20

Von Raumer JF, Vesela P, Finger F, Stampfli GM (2013) DurbachitesVaugnerites-a geodynamic marker in the central European Variscan orogen. Terra Nova 26:85-95

Von Raumer JF, Nesbor HD, Stampfli GM (2014) The plate-tectonic interpretation of the Rhenohercynian ore-sites. SDGG 85:613

Von Raumer J, Stampfli GM, Arenas R, Sánchez Martínez S (2015) Ediacaran to Cambrian oceanic rocks of the Gondwana margin and their tectonic interpretation. Int J Earth Sci (Geol Rundsch) 104:1107-1121

Walliser OH (1981) The geosynclinal development of the Rheinische Schiefergebirge (Rhenohercynian zone of the Variscides, Germany). Geol Mijnbouw 60:89-96

Werner W, Walther HW (1995) Metallogenesis. In: Dallmeyer RD, Franke W, Weber K (eds) Pre-Permian geology of central and eastern Europe. Springer, Berlin, pp 87-95

Will TM, Lee SH, Schmädicke E, Frimmel HE, Okrusch M (2015) Variscan terrane boundaries in the Odenwald-Spessart basement, Mid-German Crystalline Zone: new evidence from ocean ridge, intraplate and arc-derived metabasaltic rocks. Lithos 220-223:23-42

Zeh A, Gerdes A (2010) Baltica- and Gondwana derived sediments in the Mid-German Crystalline Rise (Central Europe): implications for the closure of the Rheic Ocean. Gondwana Res 17:254-263

Ziegler PA (1988) Evolution of the Arctic-North Atlantic and the Western Tethys. Am Ass Petrol Geol Memoir 43:198 